\title{
EFFICIENT FULL HIGHER-ORDER UNIFICATION
}

\author{
PETAR VUKMIROVIĆ, ALEXANDER BENTKAMP, AND VISA NUMMELIN
}

Vrije Universiteit Amsterdam, De Boelelaan 1111, 1081 HV Amsterdam, The Netherlands

e-mail address: \{p.vukmirovic,a.bentkamp,visa.nummelin\}@vu.nl

\begin{abstract}
We developed a procedure to enumerate complete sets of higher-order unifiers based on work by Jensen and Pietrzykowski. Our procedure removes many redundant unifiers by carefully restricting the search space and tightly integrating decision procedures for fragments that admit a finite complete set of unifiers. We identify a new such fragment and describe a procedure for computing its unifiers. Our unification procedure, together with new higher-order term indexing data structures, is implemented in the Zipperposition theorem prover. Experimental evaluation shows a clear advantage over Jensen and Pietrzykowski's procedure.
\end{abstract}

\section{INTRODUCTION}

Unification is concerned with finding a substitution that makes two terms equal, for some notion of syntactic equality. Since the invention of Robinson's first-order unification algorithm [Rob65], it has become an indispensable tool in theorem proving, logic programming, natural language processing, programming language compilation and other areas of computer science.

Many of these applications are based on higher-order formalisms and require higher-order unification. Due to its undecidability and explosiveness, the higher-order unification problem is considered one of the main obstacles on the road to efficient higher-order tools.

One of the reasons for higher-order unification's explosiveness lies in flex-flex pairs, which consist of two variable-headed terms, e.g., $F X \stackrel{?}{=} G$ a, where $F, G$, and $X$ are variables and a is a constant. Even this seemingly simple problem has infinitely many incomparable unifiers. One of the first methods designed to combat this explosion is Huet's preunification [Hue75]. Huet noticed that some logical calculi would remain complete if flex-flex pairs are not eagerly solved but postponed as constraints. If only flex-flex constraints remain, we know that a unifier must exist and we do not need to solve them. Huet's preunification has been used in many reasoning tools including Isabelle [NPW02], Leo-III [SB18], and Satallax [Bro12]. However, recent developments in higher-order theorem proving $\left[\mathrm{BBT}^{+} 19, \mathrm{BR} 19\right]$ require full unification-i.e., enumeration of unifiers even for flex-flex pairs, which is the focus of this article.

Key words and phrases: unification, higher-order logic, theorem proving, term rewriting, indexing data structures. 
Jensen and Pietrzykowski's (JP) procedure [JP76] is the best known procedure for this purpose (Section 2). Given two terms to unify, it first identifies a position where the terms disagree. Then, in parallel branches of the search tree, it applies suitable substitutions, involving a variable either at the position of disagreement or above, and repeats this process on the resulting terms until they are equal or trivially nonunifiable.

Building on the JP procedure, we designed a new procedure (Section 3) with the same completeness guarantees (Section 4). The new procedure addresses many of the issues that are detrimental to the performance of the JP procedure. First, the JP procedure does not terminate in many cases of obvious nonunifiability, e.g., for $X \stackrel{?}{=} \mathrm{f} X$, where $X$ is a non-functional variable and $f$ is a function constant. This example also shows that the JP procedure does not generalize Robinson's first-order procedure gracefully. To address this issue, our procedure detects whether a unification problem belongs to a fragment for which unification is decidable and finite complete sets of unifiers (CSUs) exist. We call algorithms that enumerate elements of the CSU for such fragments oracles. Noteworthy fragments with oracles are first-order terms, patterns [Nip93], functions-as-constructors [LM16], and a new fragment we present in Section 5. The unification procedures of Isabelle and Leo-III check whether the unification problem belongs to a decidable fragment, but we take this idea a step further by checking this more efficiently and for every subproblem arising during unification.

Second, the JP procedure computes many redundant unifiers. Consider the example $F(G \mathrm{a}) \stackrel{?}{=} F \mathrm{~b}$, where it produces, in addition to the desired unifiers $\{F \mapsto \lambda x . H\}$ and $\{G \mapsto \lambda x$. b $\}$, the redundant unifier $\{F \mapsto \lambda x . H, G \mapsto \lambda x . x\}$. The design of our procedure avoids computing many redundant unifiers, including this one. Additionally, as oracles usually return a small CSU, their integration reduces the number of redundant unifiers.

Third, the JP procedure applies more explosive rules than Huet's preunification procedure to flex-rigid pairs. To gracefully generalize Huet's procedure, we show that his rules for flex-rigid pairs suffice to enumerate CSUs if combined with appropriate rules for flex-flex pairs.

Fourth, the JP procedure repeatedly traverses the parts of the unification problem that have already been unified. Consider the problem $\mathrm{f}^{100}(G \mathrm{a}) \stackrel{?}{=} \mathrm{f}^{100}(H \mathrm{~b})$, where the exponents denote repeated application. It is easy to see that this problem can be reduced to $G \mathrm{a} \stackrel{?}{=} H \mathrm{~b}$. However, the JP procedure will wastefully retraverse the common context $\mathrm{f}^{100}[\mathrm{]}$ after applying each new substitution. Since the JP procedure must apply substitutions to the variables occurring in the common context above the position of disagreement, it cannot be easily adapted to eagerly decompose unification pairs. By contrast, our procedure is designed to decompose the pairs eagerly, never traversing a common context twice.

Last, the JP procedure does not allow to apply substitutions and $\beta$-reduce lazily. The rules of simpler procedures (e.g., first-order [HV09] and pattern unification [Nip93]) depend only on the heads of the unification pair. Thus, to determine the next step, implementations of these procedures need to substitute and $\beta$-reduce only until the heads of the current unification pair are not mapped by the substitution and are not $\lambda$-abstractions. Since the JP procedure is not based on the decomposition of unification pairs, it is unfit for optimizations of this kind. We designed our procedure to allow for this optimization.

To more efficiently find terms (in a large term set) that are unifiable with a given query term, we developed a higher-order extension of fingerprint indexing [Sch12] (Section 6). We implemented our procedure, several oracles, and the fingerprint index in the Zipperposition prover (Section 7). Since a straightforward implementation of the JP procedure already 
existed in Zipperposition, we used it as a baseline to evaluate the performance of our procedure (Section 8). The results show substantial performance improvements.

This invited article is an extended version of our FSCD-2020 paper [VBN20]. Most notable extension is the Section 4, which gives the detailed proof of completeness of our new procedure. In addition, we give proofs for all the unproved statements from the paper, expand the examples and provide more detailed explanations.

\section{BACKGROUND}

Our setting is the simply typed $\lambda$-calculus. Types $\alpha, \beta, \gamma$ are either base types or functional types $\alpha \rightarrow \beta$. By convention, when we write $\alpha_{1} \rightarrow \cdots \rightarrow \alpha_{n} \rightarrow \beta$, we assume $\beta$ to be a base type. Basic terms are free variables (denoted $F, G, H, \ldots$ ), bound variables $(x, y, z)$, and constants $(\mathrm{f}, \mathrm{g}, \mathrm{h})$. Complex terms are applications of one term to another $(s t)$ or $\lambda$-abstractions $(\lambda x . s)$. Following Nipkow [Nip93], we use these syntactic conventions to distinguish free from bound variables. Bound variables with no enclosing binder, such as $x$ in $\lambda y . x$, are called loose bound variables. We say that a term without loose bound variables is closed and a term without free variables is ground. Iterated $\lambda$-abstraction $\lambda x_{1} \ldots \lambda x_{n} . s$ is abbreviated as $\lambda \bar{x}_{n} . s$ and iterated application $\left(s t_{1}\right) \ldots t_{n}$ as $s \bar{t}_{n}$, where $n \geq 0$. Similarly, we denote a sequence of terms $t_{1}, \ldots, t_{n}$ by $\bar{t}_{n}$, omitting its length $n \geq 0$ where it can be inferred or is irrelevant. Parameters and body for any term $\lambda \bar{x}$. $s$ are defined to be $\bar{x}$ and $s$ respectively, where $s$ is not a $\lambda$-abstraction. The size of a term is inductively defined as $\operatorname{size}(F)=1 ; \operatorname{size}(x)=1 ; \operatorname{size}(\mathrm{f})=1 ; \operatorname{size}(s t)=\operatorname{size}(s)+\operatorname{size}(t) ; \operatorname{size}(\lambda x . s)=\operatorname{size}(s)+1$.

We assume the standard notions of $\alpha^{-}, \beta-, \eta$-conversions. A term is in head normal form ( hnf) if it is of the form $\lambda \bar{x} . a \bar{t}$, where $a$ is a free variable, bound variable, or a constant. In this case, $a$ is called the head of the term. By convention, $a$ and $b$ denote heads. If $a$ is a free variable, we call it a flex head; otherwise, we call it a rigid head. A term is called flex or rigid if its head is flex or rigid, respectively. By $s_{\downarrow \text { h }}$ we denote the term obtained from a term $s$ by repeated $\beta$-reduction of the leftmost outermost redex until it is in hnf. Unless stated otherwise, we view terms syntactically, as opposed to $\alpha \beta \eta$-equivalence classes. We write $s \leftrightarrow_{\alpha \beta \eta}^{*} t$ if $s$ and $t$ are $\alpha \beta \eta$-equivalent. Substitutions $(\sigma, \varrho, \theta)$ are functions from free and bound variables to terms; $\sigma t$ denotes application of $\sigma$ to $t$, which $\alpha$-renames $t$ to avoid variable capture. The composition $\varrho \sigma$ of substitutions is defined by $(\varrho \sigma) t=\varrho(\sigma t)$. A variable $F$ is mapped by $\sigma$ if $\sigma F \iota_{\alpha \beta \eta}^{*} F$. Given a substitution $\varrho$, which maps $F$ to $s$, we write $\varrho \backslash\{F \mapsto s\}$ to denote a substitution that does not map $F$ and otherwise coincides with $\varrho$. Given substitutions $\varrho$ and $\sigma$, which map disjoint sets of variables, we write $\varrho \cup \sigma$ to denote $\varrho \sigma$.

Deviating from the standard notion of higher-order subterm, we define subterms on $\beta$-reduced terms as follows: a term $t$ is a subterm of $t$ at position $\varepsilon$. If $s$ is a subterm of $u_{i}$ at position $p$, then $s$ is a subterm of $a \bar{u}_{n}$ at position i.p. If $s$ is a subterm of $t$ at position $p$, then $s$ is a subterm of $\lambda x . t$ at position 1.p. Our definition of subterm gracefully generalizes the corresponding first-order notion: $a$ is a subterm of $f a b$ at position 1 , but $f$ and $f a$ are not subterms of $f a b$. A context is a term with zero or more subterms replaced by a hole $\square$. We write $C\left[\bar{u}_{n}\right]$ for the term resulting from filling in the holes of a context $C$ with the terms $\bar{u}_{n}$ from left to right. The common context $\mathcal{C}(s, t)$ of two $\eta$-long $\beta$-reduced terms $s$ and $t$ of the same type is defined inductively as follows, assuming that $a \neq b: \mathcal{C}(\lambda x . s, \lambda y . t)=$ $\lambda x . \mathcal{C}(s,\{y \mapsto x\} t) ; \mathcal{C}\left(a \bar{s}_{m}, b \bar{t}_{n}\right)=\square ; \mathcal{C}\left(a \bar{s}_{m}, a \bar{t}_{m}\right)=a \mathcal{C}\left(s_{1}, t_{1}\right) \ldots \mathcal{C}\left(s_{m}, t_{m}\right)$.

A unification constraint $s \stackrel{?}{=} t$ is an unordered pair of two terms of the same type. A unifier of a multiset of unification constraints $E$ is a substitution $\sigma$, such that $\sigma s \leftrightarrow_{\alpha \beta \eta}^{*} \sigma t$, 
for all $s \stackrel{?}{=} t \in E$. A complete set of unifiers $(C S U)$ of $E$ is defined as a set $U$ of $E$ 's unifiers along with a set $V$ of auxiliary variables such that no $s \stackrel{?}{=} t \in E$ contains variables from $V$ and for every unifier $\varrho$ of $E$, there exists a $\sigma \in U$ and a substitution $\theta$ such that for all $X \notin V, \varrho X=\theta \sigma X$. A most general unifier $(M G U)$ is a one-element CSU. A unifier of terms $s$ and $t$ is a unifier of the singleton multiset $\{s \stackrel{?}{=} t\}$.

Remark 2.1. We use this definition of a CSU because JP's definition of a CSU, which we have adopted in our FSCD-2020 paper, is flawed. JP's definition does not employ the notion of auxiliary variables, but instead requires $\varrho X=\theta \sigma X$ for all variables mapped by $\varrho$. This is problematic because nothing prevents $\varrho$ from mapping the auxiliary variables. For example, $\sigma=\{F \mapsto \lambda x y . G y\}$ is supposed to be an MGU for $F$ a c $\stackrel{?}{=} F$ b c. But for the unifier $\varrho=\{F \mapsto \lambda x y . y, G \mapsto \lambda x$. d $\}$, without the notion of auxiliary variables, there exists no appropriate substitution $\theta$ because $\varrho G=\theta \sigma G$ requires $\theta G=\lambda x$. $\mathrm{d}$ and $\varrho F=\theta \sigma F$ requires $\theta G=\lambda x . x$.

\section{The Unification Procedure}

To unify two terms $s$ and $t$, our procedure builds a tree as follows. The nodes of the tree have the form $(E, \sigma)$, where $E$ is a multiset of unification constraints $\left\{\left(s_{1} \stackrel{?}{=} t_{1}\right), \ldots,\left(s_{n} \stackrel{?}{=} t_{n}\right)\right\}$ and $\sigma$ is the substitution constructed up to that point. The root node of the tree is ( $\{s \stackrel{?}{=} t\}$, id), where id is the identity substitution. The tree is then constructed applying the transitions listed below. The leaves of the tree are either failure nodes $\perp$ or substitutions $\sigma$. Ignoring failure nodes, the set of all substitutions in the leaves forms a complete set of unifiers for $s$ and $t$. More generally, our procedure can be used to unify a multiset $E$ of constraints by making the root of the unification tree $(E$, id).

The procedure requires an infinite supply of fresh free variables. These fresh variables must be disjoint from the variables occurring in the initial multiset $E$. Whenever a transition $(E, \sigma) \longrightarrow\left(E^{\prime}, \sigma^{\prime}\right)$ is made, all fresh variables used in $\sigma^{\prime}$ are removed from the supply and cannot be used again as fresh variables.

The transitions are parametrized by a mapping $\mathcal{P}$ that assigns a set of substitutions to a unification pair; this mapping abstracts the concept of unification rules present in other unification procedures. Moreover, the transitions are parametrized by a selection function $S$ mapping a multiset $E$ of unification constraints to one of those constraints $S(E) \in E$, the selected constraint in $E$. The transitions, defined as follows, are only applied if the grayed constraint is selected.

Succeed: $(\varnothing, \sigma) \longrightarrow \sigma$

Normalize $_{\alpha \eta}:\left(\left\{\lambda \bar{x}_{m} . s \stackrel{?}{=} \lambda \bar{y}_{n} \cdot t\right\} \uplus E, \sigma\right) \longrightarrow\left(\left\{\lambda \bar{x}_{m} . s \stackrel{?}{=} \lambda \bar{x}_{m} \cdot t^{\prime} x_{n+1} \ldots x_{m}\right\} \uplus E, \sigma\right)$ where $m \geq n, \bar{x}_{m} \neq \bar{y}_{n}$, and $t^{\prime}=\left\{y_{1} \mapsto x_{1}, \ldots, y_{n} \mapsto x_{n}\right\} t$

Normalize $_{\beta}:(\{\lambda \bar{x} . s \stackrel{?}{=} \lambda \bar{x} . t\} \uplus E, \sigma) \longrightarrow\left(\left\{\lambda \bar{x} . s_{\downarrow h} \stackrel{?}{=} \lambda \bar{x} \cdot t_{\downarrow h}\right\} \uplus E, \sigma\right)$ where $s$ or $t$ is not in hnf

Dereference: $(\{\lambda \bar{x} . F \bar{s} \stackrel{?}{=} \lambda \bar{x} . t\} \uplus E, \sigma) \longrightarrow(\{\lambda \bar{x} .(\sigma F) \bar{s} \stackrel{?}{=} \lambda \bar{x} . t\} \uplus E, \sigma)$ where none of the previous transitions apply and $F$ is mapped by $\sigma$

Fail: $\left(\left\{\lambda \bar{x} . a \bar{s}_{m} \stackrel{?}{=} \lambda \bar{x} \cdot b \bar{t}_{n}\right\} \uplus E, \sigma\right) \longrightarrow \perp$

where none of the previous transitions apply, and $a$ and $b$ are different rigid heads

Delete: $(\{s \stackrel{?}{=} s\} \uplus E, \sigma) \longrightarrow(E, \sigma)$

where none of the previous transitions apply 
OracleSucc: $(\{s \stackrel{?}{=} t\} \uplus E, \sigma) \longrightarrow(E, \varrho \sigma)$

where none of the previous transitions apply, some oracle found a finite CSU $U$ for $\sigma s \stackrel{?}{=} \sigma t$ using fresh auxiliary variables, and $\varrho \in U$; if multiple oracles found a CSU, only one of them is considered

OracleFail: $(\{s \stackrel{?}{=} t\} \uplus E, \sigma) \longrightarrow \perp$

where none of the previous transitions apply, and some oracle determined $\sigma s \stackrel{?}{=} \sigma t$ has no solutions

Decompose: $\left(\left\{\lambda \bar{x} \cdot a \bar{s}_{m} \stackrel{?}{=} \lambda \bar{x} \cdot a \bar{t}_{m}\right\} \uplus E, \sigma\right) \longrightarrow\left(\left\{s_{1} \stackrel{?}{=} t_{1}, \ldots, s_{m} \stackrel{?}{=} t_{m}\right\} \uplus E, \sigma\right)$ where none of the transitions Succeed to OracleFail apply

Bind: $(\{s \stackrel{?}{=} t\} \uplus E, \sigma) \longrightarrow(\{s \stackrel{?}{=} t\} \uplus E, \varrho \sigma)$

where none of the transitions Succeed to OracleFail apply, and $\varrho \in \mathcal{P}(s \stackrel{?}{=} t)$.

The transitions are designed so that only OracleSucc, Decompose, and Bind can introduce parallel branches in the constructed tree. OracleSucc can introduce branches using different unifiers of the CSU, Bind can introduce branches using different substitutions in $\mathcal{P}$, and Decompose can be applied in parallel with Bind.

The form of the rules OracleSucc and Bind is similar: both extend the current substitution. However, they are designed following different principles. OracleSucc solves the selected unification constraint using an efficient algorithm applicable only to certain classes of terms. On the other hand, Bind is applied to explore the whole search space for any given constraint. These rules are separated in two to make Bind applicable only if OracleSucc (or OracleFail) is not, so that possible solutions (or failures) are detected early.

Our approach is to apply substitutions and $\alpha \beta \eta$-normalize terms lazily. In this context, laziness means that the transitions Normalize $_{\alpha \eta}$, Normalize $\beta$, and Dereference partially normalize and partially apply the constructed substitution just enough to ensure that the heads are the ones we would get if the substitution was fully applied and the term was fully normalized. Additionally, the transitions that modify the constructed substitution, OracleSucc and Bind, do not apply that substitution to the unification pairs directly, but only extend it with a new binding. To support lazy dereferencing, these rules must maintain the invariant that all substitutions are idempotent. The invariant is easily preserved if the substitution $\varrho$ from the definition of OracleSucc and Bind is itself idempotent and no variable mapped by $\sigma$ occurs in $\varrho F$, for any variable $F$ mapped by $\varrho$.

The OracleSucc and OracleFail transitions invoke oracles, such as pattern unification, to compute a CSU faster, produce fewer redundant unifiers, and discover nonunifiability earlier. In some cases, addition of oracles lets the procedure terminate more often.

In the literature, oracles are usually stated under the assumption that their input belongs to the appropriate fragment. To check whether a unification constraint is inside the fragment, we need to fully apply the substitution and $\beta$-normalize the constraint. To avoid these expensive operations and enable efficient oracle integration, oracles must be redesigned to lazily discover whether the terms belong to their fragment. Most oracles contain a decomposition operation which requires only a partial application of the substitution and only partial $\beta$-normalization. If one of the constraints resulting from decomposition is not in the fragment, the original problem is not in the fragment. This allows us to detect that the problem is not in the fragment without fully applying the substitution and $\beta$-normalizing.

The core of the procedure lies in the Bind step, parameterized by the mapping $\mathcal{P}$ that determines which substitutions (called bindings) to create. The bindings are defined as follows: 
JP-style projection for $F$ : Let $F$ be a free variable of type $\alpha_{1} \rightarrow \cdots \rightarrow \alpha_{n} \rightarrow \beta$, where some $\alpha_{i}$ is equal to $\beta$ and $n>0$. Then the JP-style projection binding is

$$
F \mapsto \lambda \bar{x}_{n} . x_{i}
$$

Huet-style projection for $F$ : Let $F$ be a free variable of type $\alpha_{1} \rightarrow \cdots \rightarrow \alpha_{n} \rightarrow \beta$, where some $\alpha_{i}=\gamma_{1} \rightarrow \cdots \rightarrow \gamma_{m} \rightarrow \beta, n>0$ and $m \geq 0$. Huet-style projection is

$$
F \mapsto \lambda \bar{x}_{n} . x_{i}\left(F_{1} \bar{x}_{n}\right) \ldots\left(F_{m} \bar{x}_{n}\right)
$$

where the fresh free variables $\bar{F}_{m}$ and bound variables $\bar{x}_{n}$ are of appropriate types.

Imitation of $a$ for $F$ : Let $F$ be a free variable of type $\alpha_{1} \rightarrow \cdots \rightarrow \alpha_{n} \rightarrow \beta$ and $a$ be a free variable or a constant of type $\gamma_{1} \rightarrow \cdots \rightarrow \gamma_{m} \rightarrow \beta$ where $n, m \geq 0$. The imitation binding is

$$
F \mapsto \lambda \bar{x}_{n} . a\left(F_{1} \bar{x}_{n}\right) \ldots\left(F_{m} \bar{x}_{n}\right)
$$

where the fresh free variables $\bar{F}_{m}$ and bound variables $\bar{x}_{n}$ are of appropriate types.

Elimination for $F$ : Let $F$ be a free variable of type $\alpha_{1} \rightarrow \cdots \rightarrow \alpha_{n} \rightarrow \beta$, where $n>0$. In addition, let $1 \leq j_{1}<\cdots<j_{i} \leq n$ and $i<n$. Elimination for the sequence $\left(j_{k}\right)_{k=1}^{i}$ is

$$
F \mapsto \lambda \bar{x}_{n} \cdot G x_{j_{1}} \ldots x_{j_{i}}
$$

where the fresh free variable $G$ as well as all $x_{j_{k}}$ are of appropriate type. We call fresh variables emerging from this binding in the role of $G$ elimination variables.

Identification for $F$ and $G$ : Let $F$ and $G$ be different free variables. Furthermore, let the type of $F$ be $\alpha_{1} \rightarrow \cdots \rightarrow \alpha_{n} \rightarrow \beta$ and the type of $G$ be $\gamma_{1} \rightarrow \cdots \rightarrow \gamma_{m} \rightarrow \beta$, where $n, m \geq 0$. Then, the identification binding binds $F$ and $G$ with

$$
F \mapsto \lambda \bar{x}_{n} . H \bar{x}_{n}\left(F_{1} \bar{x}_{n}\right) \ldots\left(F_{m} \bar{x}_{n}\right) \quad G \mapsto \lambda \bar{y}_{m} . H\left(G_{1} \bar{y}_{m}\right) \ldots\left(G_{n} \bar{y}_{m}\right) \bar{y}_{m}
$$

where the fresh free variables $H, \bar{F}_{m}, \bar{G}_{n}$ and bound variables $\bar{x}_{n}, \bar{y}_{m}$ are of appropriate types. Fresh variables from this binding with the role of $H$ are called identification variables.

Iteration for $F$ : Let $F$ be a free variable of the type $\alpha_{1} \rightarrow \cdots \rightarrow \alpha_{n} \rightarrow \beta_{1}$ and let some $\alpha_{i}$ be the type $\gamma_{1} \rightarrow \cdots \rightarrow \gamma_{m} \rightarrow \beta_{2}$, where $n>0$ and $m \geq 0$. Iteration for $F$ at $i$ is

$$
F \mapsto \lambda \bar{x}_{n} . H \bar{x}_{n}\left(\lambda \bar{y} \cdot x_{i}\left(G_{1} \bar{x}_{n} \bar{y}\right) \ldots\left(G_{m} \bar{x}_{n} \bar{y}\right)\right)
$$

The free variables $H$ and $G_{1}, \ldots, G_{m}$ are fresh, and $\bar{y}$ is an arbitrary-length sequence of bound variables of arbitrary types. All new variables are of appropriate type. Due to indeterminacy of $\bar{y}$, this step is infinitely branching.

The following mapping $\mathcal{P}_{\mathrm{c}}(\lambda \bar{x} . s \stackrel{?}{=} \lambda \bar{x} . t)$ is used as the parameter $\mathcal{P}$ of the procedure:

- If the constraint is rigid-rigid, $\mathcal{P}_{\mathrm{c}}(\lambda \bar{x} . s \stackrel{?}{=} \lambda \bar{x} . t)=\varnothing$.

- If the constraint is flex-rigid, let $\mathcal{P}_{\mathrm{c}}(\lambda \bar{x} . F \bar{s} \stackrel{?}{=} \lambda \bar{x} . a \bar{t})$ be

- an imitation of $a$ for $F$, if $a$ is a constant, and

- all Huet-style projections for $F$, if $F$ is not an identification variable.

- If the constraint is flex-flex and the heads are different, let $\mathcal{P}_{\mathrm{c}}(\lambda \bar{x} . F \bar{s} \stackrel{?}{=} \lambda \bar{x} . G \bar{t})$ be

- all identifications and iterations for both $F$ and $G$, and

- all JP-style projections for non-identification variables among $F$ and $G$.

- If the constraint is flex-flex and the heads are identical, we distinguish two cases:

- if the head is an elimination variable, $\mathcal{P}_{\mathrm{c}}(\lambda \bar{x} \cdot s \stackrel{?}{=} \lambda \bar{x} \cdot t)=\varnothing$;

- otherwise, let $\mathcal{P}_{\mathrm{c}}(\lambda \bar{x} . F \bar{s} \stackrel{?}{=} \lambda \bar{x} . F \bar{t})$ be all iterations for $F$ at arguments of functional type and all eliminations for $F$. 
Comparison with the JP Procedure. The JP procedure enumerates unifiers by constructing a search tree with nodes of the form $(s \stackrel{?}{=} t, \sigma)$, where $s \stackrel{?}{=} t$ is the current unification problem and $\sigma$ is the substitution built so far. The initial node consists of the input problem and the identity substitution. Success nodes are nodes of the form $(s \stackrel{?}{=} s, \sigma)$. The set of all substitutions contained success nodes form a CSU.

To determine the child nodes of a node $(s \stackrel{?}{=} t, \sigma)$, the procedure computes the common context $C$ of $s$ and $t$, yielding term pairs $\left(s_{1}, t_{1}\right), \ldots,\left(s_{n}, t_{n}\right)$, called disagreement pairs, such that $s=C\left[s_{1}, \ldots, s_{n}\right]$ and $t=C\left[t_{1}, \ldots, t_{n}\right]$. It chooses one of the disagreement pairs $\left(s_{i}, t_{i}\right)$. Depending on the context $C$ and the chosen disagreement pair $\left(s_{i}, t_{i}\right)$, it determines a set of bindings $\mathcal{P}_{\mathrm{JP}}\left(C, s_{i}, t_{i}\right)$. For each of the bindings $\varrho \in \mathcal{P}_{\mathrm{JP}}\left(C, s_{i}, t_{i}\right)$, it creates a child node $\left((\varrho s)_{\downarrow \beta \eta} \stackrel{?}{=}(\varrho t)_{\downarrow \beta \eta}, \varrho \sigma\right)$, where $u_{\downarrow \beta \eta}$ denotes a $\beta \eta$-normal form of a term $u$.

The set of bindings $\mathcal{P}_{\mathrm{JP}}\left(C, s_{i}, t_{i}\right)$ is based on the heads of $s_{i}$ and $t_{i}$, and the free variables occurring above $s_{i}$ and $t_{i}$ in $C$. The set $\mathcal{P}_{\mathrm{JP}}\left(C, s_{i}, t_{i}\right)$ contains

- all JP-style projections for free variables that are heads of $s_{i}$ or $t_{i} i^{1}$

- an imitation of $a$ for $F$ if a free variable $F$ is the head of $s_{i}$ and a free variable or constant $a$ is the head of $t_{i}$ (or vice versa);

- all eliminations for free variables occurring above the chosen disagreement pair eliminating only the argument containing the disagreement pair;

- an identification for the heads of $s_{i}$ and $t_{i}$ if they are both free variables; and

- all iterations for the heads of $s_{i}$ and $t_{i}$ if they are free variables, and for all free variables occurring above the disagreement pair. ${ }^{2}$

Architecturally, the most noticeable difference between the JP procedure and ours is the representation of the problem: The JP procedure works on a single constraint, while our procedure maintains a multiset of constraints. At a first glance, this is a merely presentational change. However, it has consequences for termination, performance, and redundancy of the procedure.

Since the JP procedure never decomposes the common context of its only constraint, it allows iteration or elimination to be applied at a free variable above the disagreement pair, even if bindings were already applied below that free variable. This can lead to many different paths to the same unifier. In contrast, our procedure makes the decision which binding to apply to a flex-flex pair with the same head as soon as it is observed. Also, it explores the possibility of not applying a binding and decomposing the pair. In either way, the flex-flex pair is never revisited, which improves the performance and returns fewer redundant unifiers. We show that this restriction prunes the search space without influencing the completeness.

Our procedure makes the choice of child nodes based only on the heads of the chosen unification constraint. In contrast, the JP procedure tracks all the variables occurring in the common context. Thus, lazy normalization and lazy variable substitution cannot be integrated in the JP procedure a straightforward fashion. Moreover, as it does not feature a rule similar to Decompose, it always retraverses the already unified part of the problem, resulting in poor performance on deep terms.

One of the main drawbacks of the JP procedure is that it features a highly explosive, infinitely branching iteration rule. This rule is a more general version of Huet-style projection.

\footnotetext{
${ }^{1}$ In JP's formulation of projection, they explicitly mention that the projected argument must be of base type. In our presentation, this follows from $\beta$ being of base type by the convention introduced in Section 2 .

${ }^{2}$ In JP's formulation of iteration, it is not immediately obvious whether they intend to require iteration of arguments of base type. However, their Definition 2.4 [JP76] shows that they do.
} 
Its universality enables finding elements of CSU for flex-flex pairs, for which Huet-style projection does not suffice. However, the JP procedure applies iteration indiscriminately on both flex-flex and flex-rigid pairs. We discovered that our procedure remains complete if iteration is applied only on flex-flex pairs, and Huet-style projection only on flex-rigid ones. This helps our procedure terminate more often than the JP procedure. As a side-effect, the restriction of our procedure to the preunification problem is a graceful generalization of Huet procedure, with additional improvements such as oracles, lazy substitution, and lazy $\beta$-reduction.

The bindings of our procedure contain further optimizations that are absent in the JP procedure: The JP procedure applies eliminations for only one parameter at a time, yielding multiple paths to the same unifier. It applies imitations to flex-flex pairs, which we found to be unnecessary. Similarly, we found out that tracking which rules introduced which variables can avoid computing redundant unifiers: It is not necessary to apply iterations and eliminations on elimination variables, and projections on identification variables.

Examples. We present some examples that demonstrate advantages of our procedure. The displayed branches of the constructed trees are not necessarily exhaustive. We abbreviate JP-style projection as JP Proj, imitation as Imit, identification as Id, Decompose as Dc, Dereference as Dr, Normalize $\beta$ as $\mathrm{N}_{\beta}$, and Bind of a binding $x$ as $\mathrm{B}(x)$. Transitions of the JP procedure are denoted by $\Longrightarrow$. For the JP transitions we implicitly apply the generated bindings and fully normalize terms, which significantly shortens JP derivations.

Example 3.1. The JP procedure does not terminate on the problem $G \stackrel{?}{=} \mathrm{f} G$ :

$$
(G \stackrel{?}{=} \mathrm{f} G, \mathrm{id}) \stackrel{\text { Imit }}{\Longrightarrow}\left(\mathrm{f} G^{\prime} \stackrel{?}{=} \mathrm{f}^{2} G^{\prime}, \sigma_{1}\right) \stackrel{\text { Imit }}{\Longrightarrow}\left(\mathrm{f}^{2} G^{\prime \prime} \stackrel{?}{=} \mathrm{f}^{3} G^{\prime \prime}, \sigma_{2}\right) \stackrel{\text { Imit }}{\Longrightarrow} \cdots
$$

where $\sigma_{1}=\left\{G \mapsto \lambda x . f G^{\prime}\right\}$ and $\sigma_{2}=\left\{G^{\prime} \mapsto \lambda x . f G^{\prime \prime}\right\} \sigma_{1}$. By including any oracle that supports the first-order occurs check, such as the pattern oracle or the fixpoint oracle described in Section 7, our procedure gracefully generalizes first-order unification:

$$
(\{G \stackrel{?}{=} \mathrm{f} G\}, \mathrm{id}) \stackrel{\text { OracleFail }}{\longrightarrow} \perp
$$

Example 3.2. The following derivation illustrates the advantage of the Decompose rule.

$$
\begin{aligned}
& \left(\left\{\mathrm{h}^{100}(F \mathrm{a}) \stackrel{?}{=} \mathrm{h}^{100}(G \mathrm{~b})\right\}, \mathrm{id}\right) \stackrel{\mathrm{Dc}}{\longrightarrow}(\{F \mathrm{a} \stackrel{?}{=} G \mathrm{~b}\}, \mathrm{id}) \stackrel{\mathrm{B}(\mathrm{Id})}{\longrightarrow}\left(\{F \mathrm{a} \stackrel{?}{=} G \mathrm{~b}\}, \sigma_{1}\right) \\
& \stackrel{\operatorname{Dr}+\mathrm{N}_{\beta}}{\longrightarrow}\left(\left\{H \mathrm{a}\left(F^{\prime} \mathrm{a}\right) \stackrel{?}{=} H\left(G^{\prime} \mathrm{b}\right) \mathrm{b}\right\}, \sigma_{1}\right) \stackrel{\mathrm{Dc}}{\longrightarrow}\left(\left\{\mathrm{a} \stackrel{?}{=} G^{\prime} \mathrm{b}, F^{\prime} \mathrm{a} \stackrel{?}{=} \mathrm{b}\right\}, \sigma_{1}\right) \\
& \stackrel{\mathrm{B}(\text { Imit })}{\longrightarrow}\left(\left\{\mathrm{a} \stackrel{?}{=} G^{\prime} \mathrm{b}, F^{\prime} \mathrm{a} \stackrel{?}{=} \mathrm{b}\right\}, \sigma_{2}\right) \stackrel{\operatorname{Dr}+\mathrm{N}_{\beta}}{\longrightarrow}\left(\left\{\mathrm{a} \stackrel{?}{=} \mathrm{a}, F^{\prime} \mathrm{a} \stackrel{?}{=} \mathrm{b}\right\}, \sigma_{2}\right) \stackrel{\text { Delete }}{\longrightarrow}\left(\left\{F^{\prime} \mathrm{a} \stackrel{?}{=} \mathrm{b}\right\}, \sigma_{2}\right) \\
& \stackrel{\mathrm{B}(\text { Imit })}{\longrightarrow}\left(\left\{F^{\prime} \mathrm{a} \stackrel{?}{=} \mathrm{b}\right\}, \sigma_{3}\right) \stackrel{\text { Dr+N }}{\longrightarrow}\left(\{\mathrm{b} \stackrel{?}{=} \mathrm{b}\}, \sigma_{3}\right) \stackrel{\text { Delete }}{\longrightarrow}\left(\varnothing, \sigma_{3}\right) \stackrel{\text { Succeed }}{\longrightarrow} \sigma_{3}
\end{aligned}
$$

where $\sigma_{1}=\left\{F \mapsto \lambda x . H x\left(F^{\prime} x\right), G \mapsto \lambda y . H\left(G^{\prime} y\right) y\right\} ; \sigma_{2}=\left\{G^{\prime} \mapsto \lambda x\right.$. a $\} \sigma_{1}$; and $\sigma_{3}=$ $\left\{F^{\prime} \mapsto \lambda x\right.$. b $\} \sigma_{2}$. The JP procedure produces the same intermediate substitutions $\sigma_{1}$ to $\sigma_{3}$, but since it does not decompose the terms, it retraverses the common context $h^{100}[$ ] at every step to identify the contained disagreement pair:

$$
\begin{aligned}
& \left(\mathrm{h}^{100}(F \mathrm{a}) \stackrel{?}{=} \mathrm{h}^{100}(G \mathrm{~b}), \mathrm{id}\right) \stackrel{\text { ld }}{\Longrightarrow}\left(\mathrm{h}^{100}\left(H \mathrm{a}\left(F^{\prime} \mathrm{a}\right)\right) \stackrel{?}{=} \mathrm{h}^{100}\left(H\left(G^{\prime} \mathrm{b}\right) \mathrm{b}\right), \sigma_{1}\right) \\
& \stackrel{\text { Imit }}{\Longrightarrow}\left(\mathrm{h}^{100}\left(H \mathrm{a}\left(F^{\prime} \mathrm{a}\right)\right) \stackrel{?}{=} \mathrm{h}^{100}(H \mathrm{a} \mathrm{b}), \sigma_{2}\right) \stackrel{\text { Imit }}{\Longrightarrow}\left(\mathrm{h}^{100}(H \mathrm{ab}) \stackrel{?}{=} \mathrm{h}^{100}(H \mathrm{a} \mathrm{b}), \sigma_{3}\right) \stackrel{\text { Succeed }}{\Longrightarrow} \sigma_{3}
\end{aligned}
$$


Example 3.3. Even when no oracles are used, our procedure performs better than the JP procedure on small, simple problems. Consider the problem $F$ a $\stackrel{?}{=}$ a, which has a two element CSU: $\{F \mapsto \lambda x . x, F \mapsto \lambda x$. a $\}$. Our procedure terminates, finding both unifiers:

$$
\begin{aligned}
& (\{F \mathrm{a} \stackrel{?}{=} \mathrm{a}\}, \mathrm{id}) \stackrel{\text { B(JP Proj })}{\longrightarrow}(\{F \mathrm{a} \stackrel{?}{=} \mathrm{a}\},\{F \mapsto \lambda x . x\}) \stackrel{\operatorname{Dr}^{+\mathrm{N}_{\beta}}}{\longrightarrow}(\{\mathrm{a} \stackrel{?}{=} \mathrm{a}\},\{F \mapsto \lambda x . x\}) \\
& \stackrel{\text { Delete }}{\longrightarrow}(\varnothing,\{F \mapsto \lambda x . x\}) \stackrel{\text { Succeed }}{\longrightarrow}\{F \mapsto \lambda x . x\} \\
& (\{F \mathrm{a} \stackrel{?}{=} \mathrm{a}\}, \mathrm{id}) \stackrel{\text { B(Imit) }}{\longrightarrow}(\{F \mathrm{a} \stackrel{?}{=} \mathrm{a}\},\{F \mapsto \lambda x . \mathrm{a}\}) \stackrel{\operatorname{Dr}^{\prime} \mathrm{N}_{\beta}}{\longrightarrow}(\{\mathrm{a} \stackrel{?}{=} \mathrm{a}\},\{F \mapsto \lambda x . \mathrm{a}\}) \\
& \stackrel{\text { Delete }}{\longrightarrow}(\varnothing,\{F \mapsto \lambda x . \mathrm{a}\}) \stackrel{\text { Succeed }}{\longrightarrow}\{F \mapsto \lambda x . \mathrm{a}\}
\end{aligned}
$$

The JP procedure finds those two unifiers as well, but it does not terminate as it applies iterations to $F$.

Example 3.4. The search space restrictions also allow us to prune some redundant unifiers. Consider the problem $F(G \mathrm{a}) \stackrel{?}{=} F \mathrm{~b}$, where $\mathrm{a}$ and $\mathrm{b}$ are of base type. Our procedure produces only one failing branch and the following two successful branches:

$$
\begin{aligned}
& (\{F(G \mathrm{a}) \stackrel{?}{=} F \mathrm{~b}\}, \mathrm{id}) \stackrel{\mathrm{Dc}}{\longrightarrow}(\{G \mathrm{a} \stackrel{?}{=} \mathrm{b}\}, \mathrm{id}) \stackrel{\mathrm{B}(\text { Imit })}{\longrightarrow}(\{G \mathrm{a} \stackrel{?}{=} \mathrm{b}\},\{G \mapsto \lambda x . \mathrm{b}\}) \\
& \stackrel{\operatorname{Dr}+\mathrm{N}_{\beta}}{\longrightarrow}(\{\mathrm{b} \stackrel{?}{=} \mathrm{b}\},\{G \mapsto \lambda x . \mathrm{b}\}) \stackrel{\text { Delete }}{\longrightarrow}(\varnothing,\{G \mapsto \lambda x . \mathrm{b}\}) \stackrel{\text { Succeed }}{\longrightarrow}\{G \mapsto \lambda x . \mathrm{b}\} \\
& (\{F(G \mathrm{a}) \stackrel{?}{=} F \mathrm{~b}\}, \mathrm{id}) \stackrel{\mathrm{B}(\text { Elim })}{\longrightarrow}\left(\{F(G \mathrm{a}) \stackrel{?}{=} F \mathrm{~b}\},\left\{F \mapsto \lambda x \cdot F^{\prime}\right\}\right) \\
& \stackrel{\operatorname{Dr}^{\mathrm{N}}}{\longrightarrow}\left(\left\{F^{\prime} \stackrel{?}{=} F^{\prime}\right\},\left\{F \mapsto \lambda x \cdot F^{\prime}\right\}\right) \stackrel{\text { Delete }}{\longrightarrow}\left(\varnothing,\left\{F \mapsto \lambda x . F^{\prime}\right\}\right) \stackrel{\text { Succeed }}{\longrightarrow}\left\{F \mapsto \lambda x . F^{\prime}\right\}
\end{aligned}
$$

The JP procedure additionally produces the following redundant unifier:

$$
\begin{aligned}
& (F(G \mathrm{a}) \stackrel{?}{=} F \mathrm{~b}, \mathrm{id}) \stackrel{\mathrm{JP} \text { Proj }}{\Longrightarrow}(F \mathrm{a}=F \mathrm{~b},\{G \mapsto \lambda x . x\}) \\
& \stackrel{\text { Elim }}{\Longrightarrow}\left(F^{\prime}=F^{\prime},\left\{G \mapsto \lambda x . x, F \mapsto \lambda x . F^{\prime}\right\}\right) \stackrel{\text { Succeed }}{\Longrightarrow}\left\{G \mapsto \lambda x . x, F \mapsto \lambda x . F^{\prime}\right\}
\end{aligned}
$$

Moreover, the JP procedure does not terminate because an infinite number of iterations is applicable at the root. Our procedure terminates in this case since we only apply iteration binding for non base-type arguments, which $F$ does not have.

Pragmatic Variant. We structured our procedure so that most of the unification machinery is contained in the Bind step. Modifying $\mathcal{P}$, we can sacrifice completeness and obtain a pragmatic variant of the procedure that often performs better in practice. Our preliminary experiments showed that using mapping $\mathcal{P}_{\mathrm{p}}$ defined as follows is a reasonable compromise between completeness and performance:

- If the constraint is rigid-rigid, $\mathcal{P}_{\mathrm{p}}(\lambda \bar{x} . s \stackrel{?}{=} \lambda \bar{x} . t)=\varnothing$.

- If the constraint is flex-rigid, let $\mathcal{P}_{\mathrm{p}}(\lambda \bar{x} . F \bar{s} \stackrel{?}{=} \lambda \bar{x} . a \bar{t})$ be

- an imitation of $a$ for $F$, if $a$ is a constant, and

- all Huet-style projections for $F$ if $F$ is not an identification variable.

- If the constraint is flex-flex and the heads are different, let $\mathcal{P}_{\mathrm{p}}(\lambda \bar{x} . F \bar{s} \stackrel{?}{=} \lambda \bar{x} . G \bar{t})$ be

- an identification binding for $F$ and $G$, and

- all Huet-style projections for $F$ if $F$ is not an identification variable 
- If the constraint is flex-flex and the heads are identical, we distinguish two cases:

- if the head is an elimination variable, $\mathcal{P}_{\mathrm{p}}(\lambda \bar{x} . F \bar{s} \stackrel{?}{=} \lambda \bar{x} . F \bar{t})=\varnothing$;

- otherwise, let $\mathcal{P}_{\mathrm{p}}(\lambda \bar{x} . F \bar{s} \stackrel{?}{=} \lambda \bar{x} . F \bar{t})$ be the set of all eliminations bindings for $F$.

The pragmatic variant of our procedure removes all iteration bindings to enforce finite branching. Moreover, it imposes limits on the number of bindings applied, counting the applications of bindings locally, per constraint. It is useful to distinguish the Huet-style projection cases where $\alpha_{i}$ is a base type (called simple projection), which always reduces the problem size, and the cases where $\alpha_{i}$ is a functional type (called functional projection). We limit the number applications of the following bindings: functional projections, eliminations, imitations and identifications. In addition, a limit on the total number of applied bindings can be set. An elimination binding that removes $k$ arguments counts as $k$ elimination steps. Due to these limits, the pragmatic variant terminates.

To fail as soon as any of the limits is reached, the pragmatic variant employs an additional oracle. If this oracle determines that the limits are reached and the constraint is of the form $\lambda \bar{x} . F \bar{s}_{m} \stackrel{?}{=} \lambda \bar{x} . G \bar{t}_{n}$, it returns a trivial unifier - a substitution $\left\{F \mapsto \lambda \bar{x}_{m} . H, G \mapsto \lambda \bar{x}_{n} . H\right\}$, where $H$ is a fresh variable; if the limits are reached and the constraint is flex-rigid, the oracle fails; if the limits are not reached, it reports that terms are outside its fragment. The trivial unifier prevents the procedure from failing on easily unifiable flex-flex pairs.

Careful tuning of each limit optimizes the procedure for a specific class of problems. For problems originating from proof assistants, shallow unification depth usually suffices. However, hard hand-crafted problems often need deeper unification.

\section{Proof of Completeness}

Like the JP procedure, our procedure misses no unifiers:

Theorem 4.1. The procedure described in Section 3 parametrized by $\mathcal{P}_{\mathrm{c}}$ is complete, meaning that the substitutions on the leaves of the constructed tree form a CSU. More precisely, let $E$ be a multiset of constraints and let $V$ be the supply of fresh variables provided to the procedure. Then for any unifier $\varrho$ of $E$ there exists a derivation $(E, \mathrm{id}) \longrightarrow^{*} \sigma$ and a substitution $\theta$ such that for all free variables $X \notin V$, we have $\varrho X=\theta \sigma X$.

Taking a high-level view, this theorem is proved by incrementally defining states $\left(E_{j}, \sigma_{j}\right)$ and remainder substitutions $\varrho_{j}$ starting with $\left(E_{0}, \sigma_{0}\right)=(E$, id $)$ and $\varrho_{0}=\varrho$. The substitution $\varrho_{j}$ is what remains to be added to $\sigma_{j}$ to reach $\varrho_{0}$. States are defined so that the shape of the selected constraint from $E_{j}$ and the remainder substitution guide the choice of applicable transition rule. We employ a measure based on values of $E_{j}$ and $\varrho_{j}$ that decreases with each application of the rules. Therefore, eventually, we will reach the target substitution $\sigma$.

In the remaining of this section, we view terms as $\alpha \beta \eta$-equivalence classes, with the $\eta$-long $\beta$-normal form as their canonical representative. Moreover, we consider all substitutions to be fully applied. These assumptions are justified because all bindings depend only on the head of terms and hence replacing the lazy transitions Normalize ${ }_{\alpha \eta}$, Normalize ${ }_{\beta}$, and Dereference by eager counterparts only affects the efficiency but not the overall behavior of our procedure.

We now give the detailed completeness proof of Theorem 4.1. Our proof is an adaptation of the proof given by Jensen and Pietrzykowski [JP76]. Definitions and lemmas are reused, but are combined together differently to suit our procedure. We start by listing all reused definitions and lemmas from the original JP proof. The "JP" labels in their statements refer to the corresponding lemmas and definitions from the original proof. 
Definition 4.2 (JP D1.6). Given two terms $t$ and $s$ and their common context $C$, we can write $t$ as $C[\bar{t}]$ and $s$ as $C[\bar{s}]$ for some $\bar{t}$ and $\bar{s}$. The pairs $\left(s_{j}, t_{j}\right)$ are called disagreement pairs.

Definition 4.3 (JP D3.1). Given two terms $t$ and $s$, let $\lambda \bar{x} \cdot t^{\prime}$ and $\lambda \bar{y} . s^{\prime}$ be respective $\alpha$-equivalent terms such that their parameters $\bar{x}$ and $\bar{y}$ are disjoint. Then the disagreement pairs of $t^{\prime}$ and $s^{\prime}$ are called opponent pairs in $t$ and $s$.

Lemma 4.4 (JP L3.3 (1)). Let @ be a substitution and $X, Y$ be free variables such that $\varrho(X \bar{s})=\varrho(Y \bar{t})$ for some term tuples $\bar{s}$ and $\bar{t}$. Then for every opponent pair $u, v$ in $\varrho X$ and $\varrho Y$ (Definition 4.3), the head of $u$ or $v$ is a parameter of $\varrho X$ or $\varrho Y$.

In contrast to applied constants, applied variables should not be eagerly decomposed. For a constant $\mathrm{f}$, if $\mathrm{f} \bar{s} \stackrel{?}{=} \mathrm{f} \bar{t}$ has a unifier, that unifier must clearly also unify $s_{i} \stackrel{?}{=} t_{i}$ for each $i$. For a free variable $X$, a unifier of $X \bar{s} \stackrel{?}{=} X \bar{t}$ does not necessarily unify $s_{i} \stackrel{?}{=} t_{i}$. The concept of $\omega$-simplicity is a criterion on unifiers that captures some of the cases where eager decomposition is possible. Non- $\omega$-simplicity on the other hand is the main trigger of Iteration - the most explosive binding of our procedure.

Definition 4.5 (JP D3.2). An occurrence of a parameter $x$ of term $t$ in the body of $t$ is $\omega$-simple if both

(1) the arguments of $x$ are distinct and are exactly (the $\eta$-long forms of) all of the variables bound in the body of $t$, and

(2) this occurrence of $x$ is not in an argument of any parameter of $t$.

This definition is slightly too restrictive for our purposes. It is unfortunate that condition 1 requires $x$ to be applied to all instead of just some of the bound variables. The JP proof would probably work with such a relaxation, and the definition would then cover all cases where eager decomposition is possible. However, to reuse the JP lemmas, we stick to the original notion of $\omega$-simplicity and introduce the following relaxation:

Definition 4.6. An occurrence of a parameter $x$ of term $t$ in the body of $t$ is base-simple if it is $\omega$-simple or both

(1) $x$ is of base type, and

(2) this occurrence of $x$ is not in an argument of any parameter of $t$.

Lemma 4.7. Let $s$ have parameters $\bar{x}$ and a subterm $x_{j} \bar{v}$ where this occurrence of $x_{j}$ is base-simple. Then for any sequence $\bar{t}$ of (at least $j$ ) terms, the body of $t_{j}$ is a subterm of $s \bar{t}$ (after normalization) at the position of $x_{j} \bar{v}$ up to renaming of the parameters of $t_{j}$. To compare positions of $s$ and $s \bar{t}$, ignore the parameter count mismatch.

Proof. Consider the process of $\beta$-normalizing $s \bar{t}$. After substituting terms $\bar{t}$ into the body of $s$, a further reduction can only take place when some $t_{k}$ is an abstraction that gets $\operatorname{arguments}$ in $s$. The arguments $\bar{v}$ to the $x_{j}$ are distinct variables bound in the body of $s$. This follows easily from either case of the definition of base-simplicity. So $t_{j}$ is applied to the unmodified $\bar{v}$ after substituting terms $\bar{t}$ into the body of $s$. Base-simplicity also implies that $t_{j} \bar{v}$ does not occur in an argument to another $t_{k}$. Hence only the reduction of $t_{j} \bar{v}$ itself affects this subterm. The variables $\bar{v}$ match the parameter count of $t_{j}$ because we consider the $\eta$-long form of $t_{j}$; so $t_{j} \bar{v}$ reduces to the body of $t_{j}$ (modulo renaming). The position is obviously that of $x_{j} \bar{v}$.

Lemma 4.8 (JP C3.4 strengthened). Let $\varrho$ be a substitution and $X$ a free variable. If $\varrho(\lambda \bar{x} \cdot X \bar{s})=\varrho(\lambda \bar{x} \cdot X \bar{t})$ and some occurrence of the $i^{\text {th }}$ parameter of $\varrho X$ is base-simple, then $\varrho s_{i}=\varrho t_{i}$. 
Proof. By Lemma 4.7, $\varrho s_{i}$ occurs in $\varrho X(\varrho \bar{s})$ at certain position that depends only on $\varrho X$. Similarly $\varrho t_{i}$ occurs in $\varrho X(\varrho \bar{t})=\varrho X(\varrho \bar{s})$ at the same position, and hence $\varrho s_{i}=\varrho t_{i}$.

We define more properties to determine which binding to apply to a given constraint. Roughly speaking, the simple comparison form will trigger identification bindings, projectivity will trigger Huet-style projections, and simple projectivity will trigger JP-style projections.

Definition 4.9 (JP D3.4). We say that $s$ and $t$ are in simple comparison form if all $\omega$-simple heads of opponent pairs in $s$ and $t$ are distinct, and each opponent pair has an $\omega$-simple head.

Definition 4.10 (JP D3.5). A term $t$ is called projective if the head of $t$ is a parameter of $t$. If the whole body is just the parameter, then $t$ is called simply projective.

A central part of the proof is to find a suitable measure for the remaining problem size. Showing that the measure is strictly decreasing and well-founded guarantees that the procedure finds a suitable substitution in finitely many steps. We reuse the measure for remainder substitutions from JP [JP76], but embed it into a lexicographic measure to handle the decomposition steps and oracles of our procedure.

Definition 4.11 (JP D3.7). The free weight of a term $t$ is the total number of occurrences of free variables and constants in $t$. The bound weight of $t$ is the total number of occurrences (excluding occurrences $\lambda x$ ) of bound variables in $t$, but with the particular exemption: if a prefix variable $u$ has one or more $\omega$-simple occurrences in the body, then one such occurrence and its arguments are not counted. It does not matter which occurrence is not counted because in $\eta$-long form the bound weight of the arguments of an $\omega$-simple variable is the same for all occurrences of that variable.

Definition 4.12 (JP D3.8). For multisets $E$ of unification constraints and substitutions $\varrho$, our measure on pairs $(E, \varrho)$ is the lexicographic comparison of

A: the sum of the sizes of the terms in $\varrho E$

B: the sum over the free weight of $\varrho F$, for all variables $F$ mapped by $\varrho$

C: the sum over the bound weight of $\varrho F$, for all variables $F$ mapped by $\varrho$

D: the sum over the number of parameters of $\varrho F$, for all variables $F$ mapped by $\varrho$

We denote the quadruple containing these numbers as $\operatorname{ord}(E, \varrho)$. We denote the triple containing only the last three components of $\operatorname{ord}(E, \varrho)$ as ord $\varrho$. We write $<$ for the lexicographic comparison of these tuples.

The next six lemmas correspond to the bindings of our procedure and sufficient conditions for the binding to bring us closer to a given solution. This is expressed as a decrease of the ord measure of the remainder. In each of these lemmas, let $u$ be a term with a variable head $a$ and $v$ a term with an arbitrary head $b$. Let $\varrho$ be a unifier of $u$ and $v$. The conclusion, let us call it $\boldsymbol{C}$, is always the same: there exists a binding $\delta$ applicable to the problem $u \stackrel{?}{=} v$, and there exists a substitution $\varrho^{\prime}$ such that ord $\varrho^{\prime}<$ ord $\varrho$ and for all variables $X$ except the fresh variables introduced by the binding $\varrho X=\varrho^{\prime} \delta X$. For most of these lemmas, we refer to JP [JP76] for proofs. Although JP only claim $\varrho X=\varrho^{\prime} \delta X$ for variables $X$ mapped by $\varrho$, inspection of their proofs shows that the equality holds for all $X$ except the fresh variables introduced by the binding. Moreover, some of our bindings have more preconditions, yielding additional orthogonal hypotheses in our lemmas, which we address below.

Lemma 4.13 (JP L3.9). If $a=b$ is not an elimination variable and $\varrho$ a discards any of its parameters, then $\boldsymbol{C}$ by elimination. Moreover, for the elimination variable $G$ introduced by this elimination, $\varrho^{\prime} G$ discards none of its parameters and has the body of @a. 
Proof. Let $\varrho a=\lambda \bar{x} . t$ and let $\left(x_{j_{k}}\right)_{k=1}^{i}$ be the subsequence of $\bar{x}$ consisting of those variables which occur in the body $t$. It is a strict subsequence, since $\varrho a$ is assumed to discard some parameter. Since the equal heads $a=b$ of the constraint $u \stackrel{?}{=} v$ are not elimination variables, elimination for $\left(j_{k}\right)_{k=1}^{i}$ can be applied. Let $\delta=\left\{a \mapsto \lambda \bar{x} . G x_{j_{1}} \ldots x_{j_{i}}\right\}$ be the corresponding binding. Define $\varrho^{\prime}$ to be like $\varrho$ except

$$
\varrho^{\prime} a=a \quad \text { and } \quad \varrho^{\prime} G=\lambda x_{j_{1}} \ldots x_{j_{i}} . t .
$$

Obviously $\varrho^{\prime} G$ is a closed term and $\varrho X=\varrho^{\prime} \delta X$ holds for all $X \neq G$. Moreover ord $\varrho^{\prime}<$ ord $\varrho$, because free and bound weights stay the same ( $\varrho a$ and $\varrho^{\prime} G$ have the same body $t$ ) whereas the number of parameters strictly decreases. The definition of $\left(j_{k}\right)_{k=1}^{i}$ implies that $\varrho^{\prime} G$ discards none of its parameters.

Lemma 4.14 (JP L3.10). Assume that there exists a parameter $x$ of $\varrho$ a such that $x$ has a non- $\omega$-simple (Definition 4.5) occurrence in @a, which is not below another parameter, or such that $x$ has at least two $\omega$-simple occurrences in @a. Moreover, if $a=b$, to make iteration applicable, a must not be an elimination variable, and $x$ must be of functional type. Then $\boldsymbol{C}$ is achieved by iteration.

Lemma 4.15 (JP L3.11). Assume that $a$ and $b$ are different free variables. If $\varrho a$ is simply projective (Definition 4.10) and a is not an identification variable, then $\boldsymbol{C}$ by JP-style projection.

Lemma 4.16 (JP L3.12). If $\varrho a$ is not projective and $b$ is rigid, then $\boldsymbol{C}$ by imitation.

Lemma 4.17 (JP L3.13). Let $a \neq b$. Assume that $\varrho a \neq a$ and $\varrho b \neq b$ are in simple comparison form (Definition 4.9) and neither is projective. Then $\boldsymbol{C}$ by identification. Moreover, $\varrho^{\prime} H$ is not projective, where $H$ is the identification variable introduced by this application of the identification binding.

Proof. This is JP's Lemma 3.13, plus the claim that $\varrho^{\prime} H$ is not projective. Inspecting the proof of that lemma, it is obvious that $\varrho^{\prime} H$ cannot be projective because $\varrho a$ and $\varrho b$ are not projective.

Lemma 4.18. Assume that @a is projective (Definition 4.10), a is not an identification variable, and $b$ is rigid. Then $\boldsymbol{C}$ by Huet-style projection.

Proof. Since $\varrho a$ is projective, we have $\varrho a=\lambda \bar{x}_{n} . x_{k} \bar{t}_{m}$ for some $k$ and some terms $\bar{t}_{m}$. If $\varrho a$ is also simply projective, then $x_{k}$ must be non-functional and since Huet-style projection and JP-style projection coincide in that case, Lemma 4.15 applies. Hence, in the following we may assume that $\varrho a$ is not simply projective, i.e., that $m>0$.

Let $\delta$ be the Huet-style projection binding:

$$
\delta=\left\{a \mapsto \lambda \bar{x}_{n} \cdot x_{i}\left(F_{1} \bar{x}_{n}\right) \ldots\left(F_{m} \bar{x}_{n}\right)\right\}
$$

for fresh variables $F_{1}, \ldots, F_{m}$. This binding is applicable because $b$ is rigid. Let $\varrho^{\prime}$ be the same as $\varrho$ except that we set $\varrho^{\prime} a=a$ and for each $1 \leq j \leq m$ we set

$$
\varrho^{\prime} F_{j}=\lambda \bar{x}_{n} \cdot t_{j}
$$

It remains to show that ord $\varrho^{\prime}<$ ord $\varrho$. The free weight of $\varrho a$ is the same as the sum of the free weights of $\varrho^{\prime} F_{j}$ for $1 \leq j \leq m$. Thus, the free weight is the same for $\varrho$ and $\varrho^{\prime}$. The bound weight of $\varrho a$ however is exactly 1 larger than the sum of the bound weights of $\varrho^{\prime} F_{j}$ for $1 \leq j \leq m$ because of the additional occurrence of $x_{k}$ in $\varrho a$. The exemption for $\omega$-simple 
occurrences in the definition of the bound weight cannot be triggered by this occurrence of $x_{k}$ because $m>0$ and thus $x_{k}$ is not $\omega$-simple. It follows that ord $\varrho^{\prime}<$ ord $\varrho$.

We are now ready to prove the completeness theorem (Theorem 4.1).

Proof. Let $E$ be a multiset of constraints and let $V$ be the supply of fresh variables provided to our procedure. Let $\varrho$ be a unifier of $E$. We must show that there exists a derivation $(E$, id $) \longrightarrow^{*} \sigma$ and a substitution $\theta$ such that for all free variables $X \notin V$, we have $\varrho X=\theta \sigma X$.

Let $E_{0}=E$ and $\sigma_{0}=$ id. Let $\varrho_{0}=\tau \varrho$ for some renaming $\tau$, such that every free variable occurring in $\varrho_{0} E_{0}$ does not occur in $E_{0}$ and is not contained in $V$. Then $\varrho_{0}$ unifies $E_{0}$ because $\varrho$ unifies $E$ by assumption. Moreover, $\varrho_{0}=\varrho_{0} \sigma_{0}$. We proceed to inductively define $E_{j}, \sigma_{j}$ and $\varrho_{j}$ until we reach some $j$ such that $E_{j}=\varnothing$. To guarantee well-foundedness, we ensure that the measure ord $\left(\varrho_{j}, E_{j}\right)$ decreases with each step. We maintain the following invariants for all $j$ :

- $\left(E_{j}, \sigma_{j}\right) \longrightarrow\left(E_{j+1}, \sigma_{j+1}\right)$;

- $\operatorname{ord}\left(\varrho_{j}, E_{j}\right)>\operatorname{ord}\left(\varrho_{j+1}, E_{j+1}\right)$;

- $\varrho_{j}$ unifies $E_{j}$;

- $\varrho_{0} X=\varrho_{j} \sigma_{j} X$ for all free variables $X \notin V$;

- every free variable occurring in $\varrho_{j} E_{j}$ does not occur in $E_{j}$ and is not contained in $V$;

- for every identification variable $X, \varrho_{j} X$ is not projective; and

- for every elimination variable $X$, each parameter of $\varrho_{j} X$ has occurrences in $\varrho_{j} X$, all of which are base-simple.

If $E_{j} \neq \varnothing$, let $u \stackrel{?}{=} v$ be the selected constraint $S\left(E_{j}\right)$ in $E_{j}$.

First assume that an oracle is able to find a CSU for the constraint $u \stackrel{?}{=} v$. Since $\varrho_{j}$ unifies $u$ and $v$, by the definition of a CSU, the CSU discovered by the oracle contains a unifier $\delta$ of $u$ and $v$ such that there exists $\varrho_{j+1}$ and for all free variables $X$ except for the auxiliary variables of the CSU we have $\varrho_{j} X=\varrho_{j+1} \delta X$. Thus, an OracleSucc transition is applicable and yields the node $\left(E_{j+1}, \sigma_{j+1}\right)=\left(\delta\left(E_{j} \backslash\{u \stackrel{?}{=} v\}\right), \delta \sigma_{j}\right)$. Therefore we have a strict containment $\varrho_{j+1} E_{j+1} \subset \varrho_{j+1} \delta E_{j}=\varrho_{j} E_{j}$. This implies ord $\left(E_{j+1}, \varrho_{j+1}\right)<\operatorname{ord}\left(E_{j}, \varrho_{j}\right)$. It also shows that the constraints $\varrho_{j+1} E_{j+1}$ are unified when $\varrho_{j} E_{j}$ are. Since the auxiliary variables introduced by OracleSucc are fresh, they cannot occur in $E_{j}$ nor in $\sigma_{j} X$ for any $X \notin V$. Hence, we have $\varrho_{0} X=\varrho_{j} \sigma_{j} X=\varrho_{j+1} \delta \sigma_{j} X=\varrho_{j+1} \sigma_{j+1} X$ for all free variables $X \notin V$. Any free variable occurring in $\varrho_{j+1} E_{j+1}$ cannot not occur in $E_{j+1}$ and is not contained in $V$ because $\varrho_{j+1} E_{j+1} \subset \varrho_{j} E_{j}$ and the variables in $E_{j+1}=\delta\left(E_{j} \backslash\{u \stackrel{?}{=} v\}\right)$ are either variables already present in $E_{j}$ or fresh variables introduced by OracleSucc. New identification or elimination variables are not introduced; so their properties are preserved. Hence all invariants are preserved.

Otherwise we proceed by a case distinction on the form of $u \stackrel{?}{=} v$. Typically, one of the Lemmas $4.13-4.18$ is going to apply. Any one of them gives substitutions $\varrho^{\prime}$ and $\delta$ with properties that let us define $E_{j+1}=\delta E_{j}, \sigma_{j+1}=\delta \sigma_{j}$ and $\varrho_{j+1}=\varrho^{\prime}$. The problem size always strictly decreases, because these lemmas imply $\varrho_{j+1} E_{j+1}=\varrho_{j+1} \delta E_{j}=\varrho_{j} E_{j}$ and ord $\varrho_{j+1}=$ ord $\varrho^{\prime}<$ ord $\varrho_{j}$. Regarding the other invariants, the former equation guarantees that $\varrho_{j+1}$ unifies $E_{j+1}$, and $\varrho_{0} X=\varrho_{j} \sigma_{j} X=\varrho_{j+1} \delta \sigma_{j} X=\varrho_{j+1} \sigma_{j+1} X$ for all $X \notin V$ because the fresh variables introduced by the binding cannot occur in $\sigma_{j} X$ for any $X \notin V$. The conditions on variables must be checked separately when new ones are introduced. Let $a$ be the head of $u=\lambda \bar{x} \cdot a \bar{u}$ and $b$ be the head of $v=\lambda \bar{x} \cdot b \bar{v}$. Consider the following cases:

$u$ and $v$ have the same head symbol $a=b$ : 
(1) Suppose that $\varrho_{j} a$ has a parameter with non-base-simple occurrence. By one of the induction invariants, $a$ is not an elimination variable. Among all non-basesimple occurrences of parameters in $\varrho_{j} a$, choose the leftmost one, which we call $x$. This occurrence of $x$ cannot be below another parameter, because having $x$ occur in one of its arguments would make that other parameter non-base-simple, contradicting the occurrence of $x$ being leftmost. Thus $x$ is neither base-simple nor below another parameter; so $x$ is of functional type. Moreover, non-base-simplicity implies non- $\omega$-simplicity. Hence, we can apply Lemma 4.14 (iteration).

(2) Otherwise suppose that $\varrho_{j} a$ discards some of its parameters. By one of the induction invariants, $\varrho_{j} a$ is not an elimination variable. Hence Lemma 4.13 (elimination) applies. The newly introduced elimination variable $G$ satisfies the required invariants, because Lemma 4.13 guarantees that $\varrho_{j+1} G$ uses its parameters and shares the body with $\varrho_{j} a$ which by assumption of this case contains only base-simple occurrences.

(3) Otherwise every parameter of $\varrho_{j} a$ has occurrences and all of them are base-simple. We are going to show that Decompose is a valid transition and decreases $\varrho_{j} E_{j}$. By Lemma 4.8 we conclude from $\varrho_{j} u=\varrho_{j} v$ that $\varrho_{j} u_{i}=\varrho_{j} v_{i}$ for every $i$. Hence the new constraints $E_{j+1}=E_{j} \backslash\{u \stackrel{?}{=} v\} \cup\left\{u_{i} \stackrel{?}{=} v_{i} \mid\right.$ for all $\left.i\right\}$ after Decompose are unified by $\varrho_{j}$. This allows us to define $\varrho_{j+1}=\varrho_{j}$ and $\sigma_{j+1}=\sigma_{j}$. To check that $\varrho_{j+1} E_{j+1}=\varrho_{j} E_{j+1}$ is smaller than $\varrho_{j} E_{j}$ it suffices to check that constraints $\varrho_{j} u_{i} \stackrel{?}{=} \varrho_{j} v_{i}$ together are smaller than $\varrho_{j} u \stackrel{?}{=} \varrho_{j} v$. Since all parameters of $\varrho_{j} a$ have base-simple occurrences, $\varrho_{j} u_{i}$ is a subterm of $\varrho_{j} u=\lambda \bar{x} \cdot \varrho_{j} a\left(\varrho_{j} \bar{u}\right)$ by Lemma 4.7. Similarly for $\varrho_{j} v$. It follows that $\varrho_{j+1} E_{j+1}$ is smaller than $\varrho_{j} E_{j}$. Since $\varrho_{j+1}=\varrho_{j}$ and $\sigma_{j+1}=\sigma_{j}$, the other invariants are obviously preserved.

$u$ and $v$ is a flex-flex pair with different heads:

(5) First, suppose that $\varrho_{j} a$ or $\varrho_{j} b$ is simply projective (Definition 4.10). By the induction hypothesis, the simply projective head cannot be an identification variable. Thus Lemma 4.15 (JP-style projection) applies.

(6) Otherwise suppose that $\varrho_{j} a$ is projective but not simply. Then the head of $\varrho_{j} a$ is some parameter $x_{k}$. But this occurrence cannot be $\omega$-simple because it has arguments which cannot be bound above the head $x_{k}$. Thus Lemma 4.14 (iteration) applies. If $\varrho_{j} b$ is projective but not simply, the same argument applies.

(7) Otherwise suppose that $\varrho_{j} a, \varrho_{j} b$ are in simple comparison form (Definition 4.9). By one of the the induction invariants, the free variables occurring in $\varrho_{j} E_{j}$ do not occur in $E_{j}$. Thus $\varrho_{j} a \neq a$ and $\varrho_{j} b \neq b$. Then Lemma 4.17 (identification) applies.

(8) Otherwise $\varrho_{j} a, \varrho_{j} b$ are not in simple comparison form. By Lemma 4.4 and by the definition of simple comparison form, there is some opponent pair $x_{k} \bar{r}, b$ in $\varrho_{j} a$ and $\varrho_{j} b$ (after possibly swapping $u$ and $v$ ) where either the occurrence of $x_{k}$ is not $\omega$-simple (Definition 4.5) or else $x_{k}$ has another $\omega$-simple occurrence in the body of $\varrho_{j} a$. Then Lemma 4.14 (iteration) applies.

$u$ and $v$ is a flex-rigid pair: Without loss of generality, assume that $a$ is flex and $b$ is rigid.

(9) Suppose first that $\varrho_{j} a$ is projective. By one of the induction invariants, $a$ cannot be an identification variable. Thus Lemma 4.18 (Huet-style projection) applies.

(10) Otherwise $\varrho_{j} a$ is not projective. The head of $\varrho_{j} a$ must be $b$ because $b$ is rigid, and $\varrho_{j}$ unifies $u$ and $v$. Since $\varrho_{j} a$ is not projective, that means that $b$ is not a bound variable. Therefore, $b$ must be a constant. Then Lemma 4.16 (imitation) applies.

We have now constructed a run $\left(E_{0}, \sigma_{0}\right) \longrightarrow\left(E_{1}, \sigma_{1}\right) \longrightarrow\left(E_{2}, \sigma_{2}\right) \longrightarrow \cdots$ of the procedure. This run cannot be infinite because because the measure $\operatorname{ord}\left(E_{j}, \varrho_{j}\right)$ strictly decreases 
as $j$ increases. Hence, at some point we reach a $j$ such that $E_{j}=\varnothing$ and $\varrho_{0} X=\varrho_{j} \sigma_{j} X$ for all $X \notin V$. Therefore, $(E$, id $) \longrightarrow^{*}\left(\varnothing, \sigma_{j}\right) \longrightarrow \sigma_{j}$, and $\varrho X=\tau^{-1} \varrho_{j} \sigma_{j} X$ for all $X \notin V$, completing the proof.

\section{A New Decidable Fragment}

We discovered a new fragment that admits a finite CSU and a simple oracle. The oracle is based on work by Prehofer and the PT procedure [Pre95], an adaptation of the preunification procedure by Snyder and Gallier [SG89] (which itself is an adaptation of Huet's procedure). PT transforms an initial multiset of constraints $E_{0}$ by applying bindings $\varrho$. If there is a sequence $E_{0} \Longrightarrow \varrho_{1} \ldots \Longrightarrow \varrho_{n}^{\varrho_{n}} E_{n}$ such that $E_{n}$ has only flex-flex constraints, we say that PT produces a preunifier $\sigma=\varrho_{n} \ldots \varrho_{1}$ with constraints $E_{n}$. A sequence fails if $E_{n}=\perp$. As in the previous section, we consider all terms to be $\alpha \beta \eta$-equivalence classes with the $\eta$-long $\beta$-reduced form as their canonical representative. Unlike previously, in this section we view unification constraints $s \stackrel{?}{=} t$ as ordered pairs.

The following rules, however, are stated modulo orientation. The PT transition rules, adapted for our presentation style, are as follows:

Deletion: $\{s \stackrel{?}{=} s\} \uplus E \Longrightarrow$ id $E$

Decomposition: $\left\{\lambda \bar{x} \cdot a \bar{s}_{m} \stackrel{?}{=} \lambda \bar{x} \cdot a \bar{t}_{m}\right\} \uplus E \Longrightarrow \stackrel{\text { id }}{=}\left\{s_{1} \stackrel{?}{=} t_{1}, \ldots, s_{m} \stackrel{?}{=} t_{m}\right\} \uplus E$ where $a$ is rigid

Failure: $\{\lambda \bar{x} \cdot a \bar{s} \stackrel{?}{=} \lambda \bar{x} \cdot b \bar{t}\} \uplus E \Longrightarrow$ id $\perp$ where $a$ and $b$ are different rigid heads

Solution: $\{\lambda \bar{x} \cdot F \bar{x} \stackrel{?}{=} \lambda \bar{x} \cdot t\} \uplus E \Longrightarrow \varrho \varrho E$

where $F$ does not occur in $t, t$ does not have a flex head, and $\varrho=\{F \mapsto \lambda \bar{x} . t\}$

Imitation: $\left\{\lambda \bar{x} . F \bar{s}_{m} \stackrel{?}{=} \lambda \bar{x} . \mathrm{f} \bar{t}_{n}\right\} \uplus E \Longrightarrow \varrho \varrho\left(\left\{G_{1} \bar{s}_{m} \stackrel{?}{=} t_{1}, \ldots, G_{n} \bar{s}_{m} \stackrel{?}{=} t_{n}\right\} \uplus E\right)$ where $\varrho=\left\{F \mapsto \lambda \bar{x}_{m} . \mathrm{f}\left(G_{1} \bar{x}_{m}\right) \ldots\left(G_{n} \bar{x}_{m}\right)\right\}, \bar{G}_{n}$ are fresh variables of appropriate types

Projection: $\left\{\lambda \bar{x} . F \bar{s}_{m} \stackrel{?}{=} \lambda \bar{x} \cdot a \bar{t}\right\} \uplus E \Longrightarrow \varrho \varrho\left(\left\{s_{i}\left(G_{1} \bar{s}_{m}\right) \ldots\left(G_{j} \bar{s}_{m}\right) \stackrel{?}{=} a \bar{t}\right\} \uplus E\right)$ where $\varrho=\left\{F \mapsto \lambda \bar{x}_{m} . x_{i}\left(G_{1} \bar{x}_{m}\right) \ldots\left(G_{j} \bar{x}_{m}\right)\right\}, \bar{G}_{j}$ are fresh variables of appropriate types

The grayed constraints are required to be selected by a given selection function $S$. We call $S$ admissible if it selects only flex-rigid constraints, prioritizes selection of constraints applicable for Failure and Decomposition, and of descendant constraints of Projection transitions with $j=0$ (i.e., for $x_{i}$ of base type), in that order of priority. In the remainder of this section we consider only admissible selection functions, an assumption that Prehofer also makes implicitly in his thesis. Additionally, whenever we compare multisets, we use the multiset ordering defined by Dershowitz and Manna [DM79]. As above, we assume that the fresh variables are taken from an infinite supply $V$ of fresh variables that are different from the variables in the initial problem and never reused.

The following lemma states that $\mathrm{PT}$ is complete for preunification:

Lemma 5.1. Let $\varrho$ be a unifier of a multiset of constraints $E_{0}$. Then PT produces a preunifier $\sigma$ with constraints $E_{n}$, and there exists a unifier $\theta$ of $E_{n}$ such that $\varrho X=\theta \sigma X$ for all $X$ that are not contained in the supply $V$ of fresh variables.

Proof. This lemma is a refinement of Lemma 4.1.7 from Prehofer's PhD thesis [Pre95], and this proof closely follows the proof of that lemma. Compared to the lemma from Prehofer's 
thesis, our lemma additionally establishes the relationship of unifier $\theta$ of the resulting flex-flex constraint set $E_{n}$ with preunifier $\sigma$ and the target unifier $\varrho$.

We prove the lemma by induction using a well-founded measure on $\left(\varrho, E_{0}\right)$. The ordering is the lexicographic comparison of the following properties:

A: sum of the abstraction-free sizes of the terms $\varrho F$ for each variable $F$ mapped by $\varrho$

B: multiset of the sizes of constraints in $E_{0}$

Here, the abstraction-free size is inductively defined by $\operatorname{afsize}(F)=1 ; \operatorname{afsize}(x)=1$; $\operatorname{afsize}(\mathrm{f})=1 ; \operatorname{afsize}(s t)=\operatorname{afsize}(s)+\operatorname{afsize}(t) ; \operatorname{afsize}(\lambda x . s)=\operatorname{afsize}(s)$.

If $E_{0}$ consists only of flex-flex constraints, then we can take an empty transition sequence (i.e., $\sigma=\mathrm{id}$ ) and $\theta=\varrho$. Otherwise, there exists a constraint that is selected by an admissible selection function. We show that for each such constraint, there is going to be a PT transition bringing us closer to the desired preunifier.

Let $E_{0}=\{s \stackrel{?}{=} t\} \uplus E_{0}^{\prime}$. Since $s \stackrel{?}{=} t$ is selected, at least one of $s$ and $t$ must have a rigid head. We distinguish several cases based on the form of $s \stackrel{?}{=} t$ (modulo the constraint order): - $s \stackrel{?}{=} s$ : in this case, Deletion applies. This transition does not alter $\varrho$, but removes an equation obtaining $E_{1}$ which is smaller than $E_{0}$ and still unifiable by $\varrho$. By induction hypothesis, the preunifier is reachable.

- $\lambda \bar{x} \cdot a \bar{s}_{m} \stackrel{?}{=} \lambda \bar{x} \cdot b \bar{t}_{n}$, where $a$ and $b$ are rigid: since $\varrho$ is a unifier, then $a=b, n=m$, and Decomposition applies. Similarly to the above case, we conclude that the preunifier is reachable.

- $\lambda \bar{x} . F \bar{x} \stackrel{?}{=} \lambda \bar{x} . t$ where $t$ has a rigid head and $F$ does not occur in $t$ : Solution applies. Huet showed [Hue75, proof of L5.1] that in this case $\varrho=\varrho \sigma_{1}$, where $\sigma_{1}=\{F \mapsto \lambda \bar{x} . t\}$. Let $\varrho_{1}=\varrho \backslash\{F \mapsto \varrho F\}$. Then $\varrho=\varrho_{1} \sigma_{1}$. Since $\varrho$ unifies $E_{0}^{\prime}, \varrho_{1}$ unifies $E_{1}=\sigma_{1} E_{0}^{\prime}$. Clearly, $\varrho_{1}$ is smaller than $\varrho$. Now we can apply induction hypothesis on $\varrho_{1}$ and $E_{1}$, from which we obtain a sequence $E_{1} \Longrightarrow \sigma_{2}^{\sigma_{2}} \ldots \Longrightarrow^{\sigma_{n}} E_{n}, \theta$ which unifies $E_{n}$ and $\sigma^{\prime}=\sigma_{n} \ldots \sigma_{2}$, such that $\varrho_{1} X=\theta \sigma^{\prime} X$ for all $X \notin V$. Finally, it is clear that sequence $E_{0} \Longrightarrow{ }^{\sigma_{1}} E_{1} \Longrightarrow{ }^{\sigma_{2}}$ $\cdots \Longrightarrow E_{n} E_{n}, \theta$, and $\sigma=\sigma^{\prime} \sigma_{1}$ are as wanted in the lemma statement.

- $\lambda \bar{x} \cdot F \bar{s}_{m} \stackrel{?}{=} \lambda \bar{x} \cdot a \bar{t}$ where $a$ is rigid: depending on the value of $\varrho$, we can either take an Imitation or Projection step. In either case, we show that the measure reduces. Let $\varrho F=\lambda \bar{x}_{m} . b \bar{u}_{n}$ where $b$ is either a constant or a bound variable. If $b$ is a constant, we choose the Imitation step; otherwise we choose the Projection step. In either case, $\sigma_{1}=\left\{F \mapsto \lambda \bar{x}_{m} . b\left(G_{1} \bar{x}_{m}\right) \ldots\left(G_{n} \bar{x}_{m}\right)\right\}$. Then it is easy to check that $\varrho_{1}=\varrho \backslash\left\{F \mapsto \lambda \bar{x}_{m} . b \bar{u}_{n}\right\} \cup\left\{G_{1} \mapsto \lambda \bar{x}_{m} \cdot u_{1}, \ldots, G_{n} \mapsto \lambda \bar{x}_{m} . u_{n}\right\}$ unifies $E_{1}$ created as the result of imitation or projection. Clearly, $\varrho_{1}$ has smaller abstraction-free size than $\varrho$. Therefore, by the induction hypothesis we obtain the transitions $E_{1} \Longrightarrow^{\sigma_{2}} \cdots \Longrightarrow^{\sigma_{n}} E_{n}$ and substitutions $\theta$ and $\sigma^{\prime}=\sigma_{n} \ldots \sigma_{2}$, where $\varrho_{1} X=\theta \sigma^{\prime} X$ for all $X \notin V \backslash\left\{G_{1}, \ldots, G_{n}\right\}$ and $\theta$ is a unifier of $E_{n}$. Our goal is to prove $\varrho X=\theta \sigma^{\prime} \sigma_{1} X$ for all $X \notin V$. For variables $X \notin V$ that are not $F$, we have $\sigma_{1} X=X$ and $\varrho X=\varrho_{1} X$, which implies $\varrho X=\theta \sigma^{\prime} \sigma_{1} X$. For $X=F$, since $\varrho_{1}$ and $\theta \sigma^{\prime}$ agree on $G_{1}, \ldots, G_{n}$, we conclude that $\varrho F=\theta \sigma^{\prime} \sigma_{1} F$. Therefore, by taking $\sigma=\sigma^{\prime} \sigma_{1}$, and prepending $E_{0}$ to the sequence from the induction hypothesis, we show that the preunifier is reachable.

Prehofer showed that PT terminates for some classes of constraints. Those classes impose requirements on the free variables occurring in the constraints. In particular, he identified a class of terms we call strictly solid ${ }^{3}$, which requires that all arguments of free

\footnotetext{
${ }^{3}$ In Prehofer's thesis this class is described in the statement of Theorem 5.2.6.
} 
variables are either bound variables (of arbitrary type) or ground second-order terms of base type. Another important constraint is linearity: a term is called linear if it contains no repeated occurrences of free variables.

Example 5.2. Let $G$, a, and $x$ be of base type, and $F, H, \mathrm{~g}$, and $y$ be binary. Then, the term $F G$ a is not strictly solid, since $F$ is applied to a free variable $G$; similarly $H(\lambda x . x)$ a is not strictly solid as the first argument is of functional type, but it is not a bound variable; $\lambda x . F x$ a is strictly solid, but $F$ a $(\mathrm{g}(\lambda y . y \mathrm{a} a) \mathrm{a})$ is not, as the second argument of $F$ is a ground term of third order.

Prehofer's thesis states that PT terminates on $\{s \stackrel{?}{=} t\}$ if $s$ is linear, $s$ shares no free variables with $t, s$ is strictly solid, and $t$ is second-order. Together with completeness of PT for preunification, this result implies that PT procedure can be used to enumerate finitely many elements of complete set of preunifiers for this class of terms.

Prehofer focused on the preunification problem as he remarks that the resulting flex-flex pairs are "intricate" [Pre95, Sect. 5.2.2]. We discovered that these flex-flex pairs actually have an MGU, allowing us to solve the full unification problem, rather than preunification problem. Moreover, we lift the order restriction imposed by Prehofer: We identify a class of terms called solid which requires that all arguments of free variables are either bound variables (of arbitrary type) or ground (arbitrary-order) terms of base type.

Example 5.3. Consider the setting of Example 5.2. The terms $F G$ a and $H(\lambda x . x)$ a are not solid, for the same reasons they are not strictly solid. However, since the order restriction is lifted, $F$ a $(\mathrm{g}(\lambda y . y$ a a $)$ a) is solid.

In other words, we extend the above preunification decidability result for linear strictly solid terms along two axes: we create an oracle for the full unification problem and we lift the order constraints. To enumerate a CSU for a problem $E_{0}=\{s \stackrel{?}{=} t\}$, where $s$ and $t$ are solid, $s$ is linear and shares no free variables with $t$, our oracle applies the following two steps:

(1) Apply the PT procedure on $E_{0}$ to obtain a preunifier $\sigma$ with flex-flex constraints $E_{n}$.

(2) If $E_{n}$ is empty, return $\sigma$. Otherwise, choose a flex-flex constraint $u \stackrel{?}{=} v$ from $E_{n}$, and let the MGU of $u \stackrel{?}{=} v$ be $\varrho$. Then, set $E_{n}:=\varrho\left(E_{n} \backslash\{u \stackrel{?}{=} v\}\right)$ and $\sigma:=\varrho \sigma$, and repeat step 2.

To show that this oracle terminates and yields a CSU, we must prove that the PT procedure terminates on above described class of problems (Lemma 5.6). Moreover, we must show how to compute MGUs for the remaining flex-flex pairs (Lemma 5.7 and 5.8). Our results are combined together in Theorem 5.9.

Towards proving that the PT procedure terminates on above described class of problems, we first consider the corresponding matching problem. A matching problem is a unification problem $\{s \stackrel{?}{=} t\}$ where $t$ is ground. In what follows, we establish some useful properties of matching problems in which both $s$ and $t$ are solid (solid matching problems). We call the unifier of a matching problem a matcher.

Lemma 5.4. PT terminates on a solid matching problem $\{s \stackrel{?}{=} t\}$.

Proof. We show termination by designing a measure on a matching problem $E$ that decreases with each application of a PT transition (possibly followed by Decomposition or Failure steps). Our measure function compares the following properties lexicographically:

A: multiset of the sizes of right-hand sides of the constraints in $E$

B: number of free variables in $E$ 
Clearly, when applying PT transitions, the problem stays a matching problem because the applied bindings do not introduce free variables on the right-hand sides. It is also easy to check that each applied binding keeps the terms in the solid fragment. Namely, bindings for both Imitation and Projection transitions are patterns, which means that, after applying the binding, fresh free variables are applied only to bound variables or ground base-type terms. The Solution transition effectively replaces the variable with a ground term, which is obviously solid. We show each transition either trivially terminates or reduces the measure:

Deletion: A decreases.

Decomposition: A decreases.

Failure: Trivial-represents a terminal node.

Solution: This transition applies on constraints of the form $F \stackrel{?}{=} t$. This reduces A, since the constraint $F \stackrel{?}{=} t$ is removed and $F$ cannot appear on the right-hand side.

Imitation: The rule is applicable only on $\lambda \bar{x} . F \bar{s}_{m} \stackrel{?}{=} \lambda \bar{x} . f \bar{t}_{n}$. The Imitation transition replaces the constraint with $\left\{H_{i} \bar{s}_{m} \stackrel{?}{=} \bar{t}_{i} \mid 1 \leq i \leq n\right\}$. This reduces $\mathrm{A}$, as $F$ does not appear on any right-hand side.

Projection: The rule is applicable only on $\lambda \bar{x} \cdot F \bar{s}_{m} \stackrel{?}{=} \lambda \bar{x} \cdot a \bar{t}_{n}$. If $a$ is a bound variable, and we project $F$ to argument $s_{i}$ different than $a$, Failure applies and PT trivially terminates. If we project $F$ to $a$, we apply Decomposition, which reduces $A$. If $a$ is a constant, for Failure not to apply, we have to project to a base-type ground term $s_{i}$. This does not increase A, since no variables appear on right-hand sides, but removes the variable $F$ from $E$, reducing B by one.

We say $\sigma$ is a grounding substitution if for every variable $F$ mapped by $\sigma, \sigma F$ is ground.

Lemma 5.5. All unifiers produced by PT for the solid matching problem $\{s \stackrel{?}{=} t\}$ are grounding substitutions.

Proof. Closely following the proof of Lemma 5.2.5 in Prehofer's PhD thesis [Pre95], we prove our claim by induction on the length of the PT transition sequence that leads to the unifier. We know this sequence is finite by Lemma 5.4. The base case of induction, for the empty sequence, is trivial. The induction step is made using one of the following transitions:

Deletion: Trivial.

Decomposition: Trivial.

Failure: This rule is not relevant, since it will not lead to the unifier.

Solution: This rule applies the substitution $\{F \mapsto t\}$, which is grounding since $t$ is ground. Imitation: This transition applies on constraints of the form

$$
\lambda \bar{x} . F \bar{s}_{k} \stackrel{?}{=} \lambda \bar{x} . \mathrm{f} \bar{t}_{l}
$$

The binding for Imitation is $\varrho=\left\{F \mapsto \lambda \bar{x}_{k} . \mathrm{f}\left(G_{1} \bar{s}_{k}\right) \ldots\left(G_{l} \bar{s}_{k}\right)\right\}$, and reduces the problem to $\left\{G_{i} \bar{s}_{k} \stackrel{?}{=} t_{i} \mid 1 \leq i \leq l\right\}$. Since the right-hand sides are ground, any unifier $\sigma$ produced by PT must map all of the variables $G_{1}, \ldots, G_{l}$. By induction hypothesis, $\sigma G_{i}$ is ground. Therefore, $\sigma \varrho$ must map $F$ to a ground term.

Projection: This transition applies on constraints of the form

$$
\lambda \bar{x} . F \bar{s}_{k} \stackrel{?}{=} \lambda \bar{x} . t
$$

The binding for Projection is $\varrho=\left\{F \mapsto \lambda \bar{x}_{k} \cdot x_{i}\left(G_{1} \bar{x}_{k}\right) \ldots\left(G_{j} \bar{x}_{k}\right)\right\}$, and reduces the problem to $\left\{s_{i}\left(G_{1} \bar{s}_{k}\right) \ldots\left(G_{j} \bar{s}_{k}\right) \stackrel{?}{=} a \bar{t}_{l}\right\}$. Since the right-hand side is ground, any unifier $\sigma$ produced by PT must map all of the variables $G_{1}, \ldots, G_{j}$. By induction hypothesis, $\sigma G_{i}$ is ground. Therefore, $\sigma \varrho$ must map $F$ to a ground term. 
Lemma 5.6. If $s$ and $t$ are solid, $s$ is linear and shares no free variables with $t$, then PT terminates for the preunification problem $\{s \stackrel{?}{=} t\}$, and all remaining flex-flex constraints are solid.

Proof. This lemma is a modification of Lemma 5.2.1 and Theorem 5.2.6 from Prehofer's $\mathrm{PhD}$ thesis [Pre95]. Correspondingly, the following proof closely follows the proofs for these two lemmas. We also adopt the notion of an isolated variable from Prehofer's thesis: a variable is isolated in a multiset $E$ of constraints if it appears exactly once in $E$.

First, similarly to the proof of previous lemma we conclude each transition maintains the condition that the terms remain solid. Second, we have to show that variables on left-hand sides remain isolated. For Imitation and Projection rules, the preservation of this invariant is obvious. Later, we also show that Solution preserves it. Third, since $s$ and $t$ share no variables, and $s$ is linear, no rule can introduce a variable from the right-hand side to the left-hand side.

To prove termination of $\mathrm{PT}$, we devise a measure that decreases with each application of a PT transition. The measure lexicographically compares the following properties:

A: number of occurrences of constant symbols and bound variables on left-hand sides that are not below free variables

B: number of free variables on right-hand sides

C: multiset of the sizes of right-hand sides

We show that each transition either trivially terminates or reduces the measure:

Deletion: A does not increase and at least one of A or B reduces.

Decomposition: A reduces.

Failure: Trivial.

Solution: This rule applies in two cases:

- $F \stackrel{?}{=} t$ : since $F$ is isolated, $\mathrm{A}$ is unchanged, $\mathrm{B}$ is not increased, and $\mathrm{C}$ reduces. All variables on left-hand sides remain isolated since they are unaffected by this substitution.

- $t \stackrel{?}{=} F$ : since $F$ does not occur on any left-hand side and the head of $t$ must be a constant or bound variable (otherwise the rule would not be applicable), $A$ reduces. Even though $t$ might contain some free variables and $F$ can have multiple occurrences on right-hand sides, all free variables on left-hand sides remain isolated. Namely, all free variables in $t$ occur exactly once in the multiset, and since $t \stackrel{?}{=} F$ is removed, all of them either disappear or end up on right-hand sides.

Imitation: We distinguish the following two forms of the selected constraint:

- $\lambda \bar{x} . F \bar{v}_{n} \stackrel{?}{=} \lambda \bar{x} . \mathrm{f} \bar{u}_{m}$ : We replace this constraint by $\left\{H_{i} \bar{v}_{n} \stackrel{?}{=} u_{i} \mid 1 \leq i \leq m\right\}$ and apply the Imitation binding $F \mapsto \lambda \bar{x}_{n} . \mathrm{f}\left(H_{1} \bar{x}_{n}\right) \ldots\left(H_{m} \bar{x}_{n}\right)$. As $F$ is isolated, A and B do not increase. Since $F$ is isolated, it does not occur on any right-hand side, and hence $\mathrm{C}$ decreases.

- $\lambda \bar{x} . \mathrm{f} \bar{u}_{m} \stackrel{?}{=} \lambda \bar{x} . F \bar{v}_{n}$ : applying the Imitation transition as above will reduce A since $F$ cannot appear on any left-hand side.

Projection: Similarly to the previous transition rule, we have two cases:

- $\lambda \bar{x} \cdot F \bar{v}_{n} \stackrel{?}{=} \lambda \bar{x} \cdot a \bar{u}_{m}$ : if $a$ is a bound variable, then for Failure not to be applicable afterwards, we have to project $F$ onto argument $v_{i}$ equal to $a$. Then we proceed like for Imitation. If $a$ is not a bound variable, then we have to project to some base-type term $v_{j}$ (otherwise Failure would be applicable afterwards). This reduces the problem to $\lambda \bar{x} \cdot v_{j} \stackrel{?}{=} \lambda \bar{x} \cdot a \bar{u}_{m}$. This is a solid matching problem, whose solutions computed by PT are grounding substitutions (see Lemma 5.5). Applying one of those solutions will eliminate all the free variables in $\lambda \bar{x} . a \bar{u}_{m}$. Since PT is parametrized by an admissible selection function, we know that there are no constraints descending from a simple projection in $E$ since 
the constraint $\lambda \bar{x} . F \bar{v}_{n} \stackrel{?}{=} \lambda \bar{x} . a \bar{u}_{m}$ was chosen, which, due to solidity restrictions, cannot be such a descendant. Therefore, we know that PT will transform the descendants of the matching problem $\lambda \bar{x} \cdot v_{j} \stackrel{?}{=} \lambda \bar{x} . a \bar{u}_{m}$ until either Failure is observed (making PT trivially terminating) or until no descendant exists and the grounding matcher is computed (see Lemmas 5.5 and 5.4). This results in removal of the original constraint $\lambda \bar{x} . F \bar{v}_{n} \stackrel{?}{=}$ $\lambda \bar{x} . a \bar{u}_{m}$ and application of the computed grounding matcher, which will either remove all the free variables in the right-hand side of the constraint (not increasing $\mathrm{A}$ and reducing $\mathrm{B}$ ) or not increasing $\mathrm{A}$ and $\mathrm{B}$, reduce $\mathrm{C}$ if no free variables occur in the right-hand side.

- $\lambda \bar{x} . a \bar{v}_{n} \stackrel{?}{=} \lambda \bar{x} . F \bar{u}_{m}$ : if $a$ is a bound variable, projecting $F$ onto argument $u_{i}$ will either enable application of Decomposition as the next step reducing A, or it will result in Failure, trivially terminating. If $a$ is a constant, then projecting $F$ onto some $u_{j}$ will either yield Failure or enable Decomposition, reducing A.

Enumerating a CSU for a solid flex-flex pair may seem as hard as for any other flex-flex pair; however, the following two lemmas show that solid pairs admit an MGU:

Lemma 5.7. The unification problem $\left\{\lambda \bar{z} \cdot F \bar{s}_{m} \stackrel{?}{=} \lambda \bar{z} \cdot F \bar{s}_{m}^{\prime}\right\}$, where both terms are solid, has an $M G U$ of the form $\sigma=\left\{F \mapsto \lambda \bar{x}_{m} . G x_{j_{1}} \ldots x_{j_{r}}\right\}$ where $G$ is an auxiliary variable, and $1 \leq j_{1}<\cdots<j_{r} \leq m$ are exactly those indices $j_{i}$ for which $s_{j_{i}}=s_{j_{i}}^{\prime}$.

Proof. Let $\varrho$ be a unifier for the given unification problem. Let $\lambda \bar{x} . u=\varrho F$. Take an arbitrary subterm of $u$ whose head is a bound variable $x_{i}$. If $x_{i}$ is of function type, it corresponds to either $s_{i}$ or $s_{i}^{\prime}$ which, due to solidity restrictions, has to be a bound variable. Furthermore, since $\varrho$ is a unifier, $s_{i}$ and $s_{i}^{\prime}$ have to be the syntactically equal. Similarly, if $x_{i}$ is of base type, it corresponds to two ground terms $s_{i}$ and $s_{i}^{\prime}$ which have to be syntactically equal. We conclude that $\varrho$ can use variables from $\bar{x}_{n}$ only if they correspond to syntactically equal terms. Therefore, there is a substitution $\theta$ such that $\varrho X=\theta \sigma X$ for all $X \neq G$. Due to arbitrary choice of $\varrho$, we conclude that $\sigma$ is an MGU.

Lemma 5.8. Let $\left\{\lambda \bar{x} . F \bar{s}_{m} \stackrel{?}{=} \lambda \bar{x} . F^{\prime} \bar{s}_{m^{\prime}}^{\prime}\right\}$ be a solid unification problem where $F \neq F^{\prime}$. By Lemma 5.4, there exists a finite $C S U\left\{\sigma_{i}^{1}, \ldots, \sigma_{i}^{k_{i}}\right\}$ of the problem $\left\{s_{i} \stackrel{?}{=} H_{i} \bar{s}_{m^{\prime}}^{\prime}\right\}$, where $H_{i}$ is a fresh free variable. Let $\lambda \bar{y}_{m^{\prime}} . s_{i}^{j}=\lambda \bar{y}_{m^{\prime}} \cdot \sigma_{i}^{j}\left(H_{i}\right) \bar{y}_{m^{\prime}}$. Similarly, also by Lemma 5.4, there exists a finite $\operatorname{CSU}\left\{\tilde{\sigma}_{i}^{1}, \ldots, \tilde{\sigma}_{i}^{l_{i}}\right\}$ of the problem $\left\{s_{i}^{\prime} \stackrel{?}{=} \tilde{H}_{i} \bar{s}_{m}\right\}$, where $\tilde{H}_{i}$ is a fresh free variable. Let $\lambda \bar{x}_{m} . s_{i}^{\prime j}=\lambda \bar{x}_{m} . \tilde{\sigma}_{i}^{j}\left(\tilde{H}_{i}\right) \bar{x}_{m}$. Let $Z$ be a fresh free variable. An $M G U \sigma$ for the given problem is

$$
\begin{aligned}
& F \mapsto \lambda \bar{x}_{m} \cdot Z \underbrace{x_{1} \ldots x_{1}}_{k_{1} \text { times }} \ldots \underbrace{x_{m} \ldots x_{m}}_{k_{m} \text { times }} s_{1}^{\prime 1} \ldots s_{1}^{\prime l_{1}} \ldots s_{m^{\prime}}^{\prime 1} \ldots s_{m^{\prime}}^{\prime l_{m^{\prime}}} \\
& F^{\prime} \mapsto \lambda \bar{y}_{m^{\prime}} \cdot Z s_{1}^{1} \ldots s_{1}^{k_{1}} \ldots s_{m}^{1} \ldots s_{m}^{k_{m}} \underbrace{y_{1} \ldots y_{1}}_{l_{1} \text { times }} \ldots \underbrace{y_{m^{\prime}} \ldots y_{m^{\prime}}}_{l_{m^{\prime}} \text { times }}
\end{aligned}
$$

where the auxiliary variables are $H_{1}, \ldots, H_{m}, \tilde{H}_{1}, \ldots \tilde{H}_{m^{\prime}}, Z$ and all auxiliary variables associated with the above CSUs.

Proof. Let $\varrho$ be an arbitrary unifier for the problem $\lambda \bar{x} . F \bar{s}_{m} \stackrel{?}{=} \lambda \bar{x} \cdot F^{\prime} \bar{s}_{m^{\prime}}^{\prime}$. We prove $\sigma$ is an MGU by showing that there exists a substitution $\theta$ such that $\varrho X=\theta \sigma X$ for all non-auxiliary variables $X$. We can focus only on $X \in\left\{F, F^{\prime}\right\}$ because all other non-auxiliary variables do neither appear in the original problem nor in $\sigma F$ or $\sigma F^{\prime}$; so we can simply define $\theta X=\varrho X$.

Let $\lambda \bar{x}_{m} \cdot u=\varrho F$ and $\lambda \bar{y}_{m^{\prime}} \cdot u^{\prime}=\varrho F^{\prime}$, where the bound variables $\bar{x}_{m}$ and $\bar{y}_{m^{\prime}}$ have been $\alpha$-renamed apart. We also assume that the names of variables bound inside $u$ and $u^{\prime}$ are 
$\alpha$-renamed so that they are different from $\bar{x}_{m}$ and $\bar{y}_{m^{\prime}}$. Finally, bound variables from the definition of $\sigma$ have been $\alpha$-renamed to match $\bar{x}_{m}$ and $\bar{y}_{m^{\prime}}$. We define $\theta$ to be the substitution

$$
\theta=\left\{Z \mapsto \lambda z_{1}^{1} \ldots z_{1}^{k_{1}} \ldots z_{m}^{1} \ldots z_{m}^{k_{m}} w_{1}^{1} \ldots w_{1}^{l_{1}} \ldots w_{m^{\prime}}^{1} \ldots w_{m^{\prime}}^{l_{m^{\prime}}} \operatorname{diff}\left(u, u^{\prime}\right)\right\}
$$

where $\operatorname{diff}\left(v, v^{\prime}\right)$ is defined recursively as

$$
\begin{aligned}
& \operatorname{diff}\left(\lambda x . v, \lambda y \cdot v^{\prime}\right)=\lambda x \cdot \operatorname{diff}\left(v,\{y \mapsto x\} v^{\prime}\right) \\
& \operatorname{diff}\left(a \bar{v}_{n}, a \bar{v}_{n}^{\prime}\right) \quad=a \operatorname{diff}\left(v_{1}, v_{1}^{\prime}\right) \ldots \operatorname{diff}\left(v_{n}, v_{n}^{\prime}\right) \\
& \operatorname{diff}\left(x_{i}, v^{\prime}\right) \quad=z_{i}^{k} \text {, if } v^{\prime}=s_{i}^{k} \\
& \operatorname{diff}\left(v, y_{i}\right) \quad=w_{i}^{l} \text {, if } v=s^{\prime l} \\
& \operatorname{diff}\left(x_{i} \bar{v}_{n}, y_{j} \bar{v}_{n}^{\prime}\right)=z_{i}^{k} \operatorname{diff}\left(v_{1}, v_{1}^{\prime}\right) \ldots \operatorname{diff}\left(v_{n}, v_{n}^{\prime}\right), \text { if } y_{j}=s_{i}^{k}
\end{aligned}
$$

From diff's definition it is clear that there are terms $v, v^{\prime}$ for which it is undefined. However, we will show that for each $u$ and $u^{\prime}$ that are bodies of bindings from a unifier $\varrho$, diff is defined and has the desired property. In equations 5.3, 5.4, and 5.5, if there are multiple numbers $k$ or $l$ that fulfill the condition, choose an arbitrary one. We need to show that $\varrho F=$ $\theta \sigma F$ and $\varrho F^{\prime}=\theta \sigma F^{\prime}$. By the definitions of $u, u^{\prime}, \theta$ and $\sigma$ and $\beta$-reduction, this is equivalent to

$$
\begin{aligned}
\lambda \bar{x}_{m} \cdot u & =\lambda \bar{x}_{m} \cdot\left\{z_{i}^{k} \mapsto x_{i}, w_{i}^{l} \mapsto s^{\prime}{ }_{i} \text { for all } k, l, i\right\} \operatorname{diff}\left(u, u^{\prime}\right) \\
\lambda \bar{y}_{m^{\prime}} \cdot u^{\prime} & =\lambda \bar{y}_{m^{\prime}} \cdot\left\{z_{i}^{k} \mapsto s_{i}^{k}, w_{i}^{l} \mapsto y_{i} \text { for all } k, l, i\right\} \operatorname{diff}\left(u, u^{\prime}\right)
\end{aligned}
$$

We will show by induction that for any $\lambda \bar{x}_{m} . v, \lambda \bar{y}_{m} \cdot v^{\prime}$ such that

$$
\left\{x_{1} \mapsto s_{1}, \ldots, x_{m} \mapsto s_{m}\right\} v=\left\{y_{1} \mapsto s_{1}^{\prime}, \ldots, y_{m^{\prime}} \mapsto s_{m^{\prime}}^{\prime}\right\} v^{\prime}
$$

we have

$$
\begin{aligned}
v & =\left\{z_{i}^{k} \mapsto x_{i}, w_{i}^{l} \mapsto s_{i}^{\prime l} \text { for all } k, l, i\right\} \operatorname{diff}\left(v, v^{\prime}\right) \\
v^{\prime} & =\left\{z_{i}^{k} \mapsto s_{i}^{k}, w_{i}^{l} \mapsto y_{i} \text { for all } k, l, i\right\} \operatorname{diff}\left(v, v^{\prime}\right)
\end{aligned}
$$

The equation $(\star)$ holds for $v=u$ and $v^{\prime}=u^{\prime}$ because $\varrho$ is a unifier of $\lambda \bar{x} \cdot F \bar{s}_{m} \stackrel{?}{=} \lambda \bar{x} \cdot F^{\prime} \bar{s}_{m^{\prime}}^{\prime}$. Therefore, once we have shown that $(\star)$ implies $(\dagger)$, we know that $(\dagger)$ holds for $v=u$ and $v^{\prime}=u^{\prime}$ and we are done.

We prove that $(\star)$ implies $(\dagger)$ by induction on the size of $v$ and $v^{\prime}$. We consider the following cases:

$v=\lambda x . v_{1}$ : For $(\star)$ to hold, $v$ and $v^{\prime}$ must be of the same type. Therefore, the $\lambda$-prefixes of their $\eta$-long representatives must have the same length and we can apply equation 5.1. By the induction hypothesis, $(\dagger)$ holds.

$v=x_{i}$ : In this case, $\left\{x_{1} \mapsto s_{1}, \ldots, x_{m} \mapsto s_{m}\right\} v=s_{i}$. Since $(\star)$ holds, $v^{\prime}$ must be an instance of a unifier from the CSU of $s_{i}=H_{i} \bar{s}_{m^{\prime}}^{\prime}$. However, since $s_{i}$ and all terms in $\bar{s}_{m^{\prime}}^{\prime}$ are ground, $\lambda \bar{y}_{m} \cdot v^{\prime}=\sigma_{i}^{k}\left(H_{i}\right)$, for some $k$. Then, $\operatorname{diff}\left(x_{i}, v^{\prime}\right)=z_{i}^{k}$, and it is easy to check that $(\dagger)$ holds. $v=x_{i} \bar{v}_{n}, n>0$ : In this case, $x_{i}$ is mapped to $s_{i}$ which, due to solidity restrictions, has to be a functional bound variable. Since $(\star)$ holds, we conclude that the head of $\left\{y_{1} \mapsto s_{1}^{\prime}, \ldots, y_{m^{\prime}} \mapsto s_{m^{\prime}}^{\prime}\right\} v^{\prime}$ must be $s_{j}^{\prime}$, such that $s_{j}^{\prime}=s_{i}$; this also means that $v^{\prime}=y_{j} \bar{v}_{n}^{\prime}$. Therefore, it is easy to check that some $\tau=\left\{H_{i} \mapsto \lambda \bar{y}_{m^{\prime}} . y_{j}\right\}$ is a matcher for the problem $s_{i}=H_{i} \bar{s}_{m^{\prime}}^{\prime}$. For some $k, \sigma_{i}^{k}=\tau$, i.e., $\operatorname{diff}\left(v, v^{\prime}\right)=z_{i}^{k} \operatorname{diff}\left(v_{1}, v_{1}^{\prime}\right) \ldots \operatorname{diff}\left(v_{n}, v_{n}^{\prime}\right)$. By induction hypothesis, we get that $(\dagger)$ holds. 
$v=a \bar{v}_{n}:$ In the remaining cases $a$ is either a free variable, a loose bound variable different than $x_{1}, \ldots, x_{m}$, or a constant. If $a$ is a free variable or a loose bound variable different than $x_{1}, \ldots, x_{m}$, then $v^{\prime}=a{\overline{v^{\prime}}}_{n}$, since $(\star)$ holds, all of $\bar{s}_{m^{\prime}}^{\prime}$ are ground, and bound variables different than $x_{1}, \ldots, x_{m}$ and $y_{1}, \ldots, y_{m^{\prime}}$ are renamed to match by equation 5.1 . By the induction hypothesis and by equation 5.2 , we obtain $(\dagger)$. If $a$ is a constant, we consider two cases: either $v^{\prime}=a{\overline{v^{\prime}}}_{n}$, which allows us to apply the induction hypothesis and obtain $(\dagger)$ as above, or $v^{\prime}=y_{j}{\overline{v^{\prime}}}_{m}$. Since $a$ is a constant, $y_{j}$ cannot be a functional bound variable, since then it would be mapped to $s_{j}^{\prime}$, which due to solidity restrictions also has to be a functional bound variable and $(\star)$ would not hold. Therefore $v^{\prime}=y_{j}$. In this case, we proceed as in the case $v=x_{i}$ with the roles of $v$ and $v^{\prime}$ swapped.

Theorem 5.9. Let $s$ and $t$ be solid terms that share no free variables, and let $s$ be linear. Then the unification problem $\{s \stackrel{?}{=} t\}$ has a finite $C S U$.

Proof. By Lemma 5.6, PT terminates on $\{s \stackrel{?}{=} t\}$ with a finite set of preunifiers $\sigma$, each associated with a multiset $E$ of solid flex-flex pairs.

An MGU $\delta_{E}$ of the remaining multiset $E$ of solid flex-flex constraints can be found as follows. Choose one constraint $(u \stackrel{?}{=} v) \in E$ and determine an MGU $\varrho$ for it using Lemma 5.7 or 5.8. Then the set $\varrho(E \backslash\{u \stackrel{?}{=} v\})$ also contains only solid flex-flex constraints, and we iterate this process by choosing a constraint from $\varrho(E \backslash\{u \stackrel{?}{=} v\})$ next until there are no constraints left, eventually yielding an MGU $\varrho^{\prime}$ of $\varrho(E \backslash\{u \stackrel{?}{=} v\})$. Finally, we obtain the MGU $\delta_{E}=\varrho^{\prime} \varrho$ of $E$.

Let $U=\left\{\delta_{E} \sigma \mid\right.$ PT produces preunifier $\sigma$ with constraints $\left.E\right\}$. By termination of PT, $U$ is finite. We show that $U$ is a CSU. Let $\varrho$ be an arbitrary unifier for $\{s \stackrel{?}{=} t\}$. By Lemma 5.1, PT produces a preunifier $\sigma$ with flex-flex constraints $E$ such that there is a unifier $\theta$ of $E$ and $\varrho X=\theta \sigma X$ for all $X$ not contained in the supply of fresh variables $V$. Since $\delta_{E}$ is an MGU of $E$, assuming that we use variables from $V$ in the role of the auxiliary variables, there exists a substitution $\theta^{\prime}$ such that $\theta X=\theta^{\prime} \delta_{E} X$ for all $X \notin V$. If we make sure that we never reuse fresh variables and that the supply $V$ does not contain any variables from the initial problem, it follows that $\varrho X=\theta^{\prime} \delta_{E} \sigma X$ for all $X \notin V$. Therefore, $U$ is a CSU.

The proof of Theorem 5.9 provides an effective way to calculate a CSU using PT and the results of Lemmas 5.7 and 5.8.

Example 5.10. Let $\{F(\mathrm{fa}) \stackrel{?}{=} \mathrm{ga}(G \mathrm{a})\}$ be the unification problem to solve. Projecting $F$ onto the first argument will lead to a nonunifiable problem, so we perform imitation of $\mathrm{g}$ building a binding $\sigma_{1}=\left\{F \mapsto \lambda x \cdot \mathrm{g}\left(F_{1} x\right)\left(F_{2} x\right)\right\}$. This yields the problem $\left\{F_{1}(\mathrm{f} \mathrm{a}) \stackrel{?}{=} \mathrm{a}\right.$, $\left.F_{2}(\mathrm{f} a) \stackrel{?}{=} G \mathrm{a}\right\}$. Again, we can only imitate a for $F_{1}$-building a new binding $\sigma_{2}=\left\{F_{1} \mapsto\right.$ $\lambda x$. a $\}$. Finally, this yields the problem $\left\{F_{2}(\mathrm{f} a) \stackrel{?}{=} G \mathrm{a}\right\}$. According to Lemma 5.8, we find CSUs for the problems $J_{1} \mathrm{a}=\mathrm{fa}$ and $I_{1}(\mathrm{fa}) \stackrel{?}{=}$ a using PT. The latter problem has a singleton CSU $\left\{I_{1} \mapsto \lambda x . \mathrm{a}\right\}$, whereas the former has a CSU containing $\left\{J_{1} \mapsto \lambda x . \mathrm{f} x\right\}$ and $\left\{J_{1} \mapsto \lambda x . \mathrm{fa}\right\}$. Combining these solutions, we obtain an MGU $\sigma_{3}=\left\{F_{2} \mapsto \lambda x . H x x\right.$ a, $G \mapsto$ $\lambda x . H(\mathrm{fa})(\mathrm{f} x) x\}$ for $F_{2}(\mathrm{fa}) \stackrel{?}{=} G$ a. Finally, we get the MGU $\sigma=\sigma_{3} \sigma_{2} \sigma_{1}=\{F \mapsto$ $\lambda x$.g a $(H x x \mathrm{a}), G \mapsto \lambda x . H(\mathrm{fa})(\mathrm{f} x) x\}$ of the original problem. For brevity, we omitted the intermediate bindings of auxiliary variables in $\sigma$.

Example 5.10 shows that the solid fragment is useful for automatic theorem provers based on $\lambda$-superposition $\left[\mathrm{BBT}^{+} 19\right]$. When the solid oracle is used, superposing from $F(\mathrm{fa})$ into g a $(G$ a) yields a single clause; without it, our procedure does not terminate. 
Small examples that violate conditions of Theorem 5.9 and admit only infinite CSUs can be found easily. The problem $\{\lambda x . F(\mathrm{f} x) \stackrel{?}{=} \lambda x . \mathrm{f}(F x)\}$ violates variable distinctness and is a well-known example of a problem with only infinite CSUs. Similarly, $\lambda x \cdot \mathrm{g}(F(\mathrm{f} x)) F \stackrel{?}{=}$ $\lambda x \cdot \mathrm{g}(\mathrm{f}(G x)) G$, which violates linearity, reduces to the previous problem. Only ground arguments to free variables are allowed because $\{F X \stackrel{?}{=} G$ a $\}$ has only infinite CSUs. Finally, it is crucial that functional arguments to free variables are only bound variables: the problem $\{\lambda y . X(\lambda x . x) y \stackrel{?}{=} \lambda y . y\}$ has only infinite CSUs.

\section{An EXtension of Fingerprint Indexing}

A fundamental building block for almost all automated reasoning tools is the operation of retrieving term pairs that satisfy certain conditions, e.g., unifiable terms, instances or generalizations. Indexing data structures are used to implement this operation efficiently. If the data structure retrieves precisely the terms that satisfy the condition, it is called perfect.

Higher-order indexing has received little attention compared to its first-order counterpart. However, recent research in higher-order theorem proving increased the interest in higherorder indexing [LS16, BR19]. A fingerprint index [Sch12, VBCS19] is an imperfect index based on the idea that the skeleton of the term consisting of all non-variable positions is not affected by substitutions. Therefore, we can easily determine that the terms are not unifiable (or matchable) if they disagree on a fixed set of sample positions.

More formally, when we sample an untyped first-order term $t$ on a sample position $p$, the generic fingerprinting function gfpf distinguishes four possibilities:

$$
\operatorname{gfpf}(t, p)= \begin{cases}\mathrm{f} & \text { if }\left.t\right|_{p} \text { has a symbol head } \mathrm{f} \\ \mathrm{A} & \text { if }\left.t\right|_{p} \text { is a variable } \\ \mathrm{B} & \text { if }\left.t\right|_{q} \text { is a variable for some proper prefix } q \text { of } p \\ \mathrm{~N} & \text { otherwise }\end{cases}
$$

We define the fingerprinting function $\operatorname{fp}(t)=\left(\operatorname{gfpf}\left(t, p_{1}\right), \ldots, \operatorname{gfpf}\left(t, p_{n}\right)\right)$, based on a fixed tuple of positions $\bar{p}_{n}$. Determining whether two terms are compatible for a given retrieval operation reduces to checking their fingerprints' componentwise compatibility. The following matrices determine the compatibility for retrieval operations:
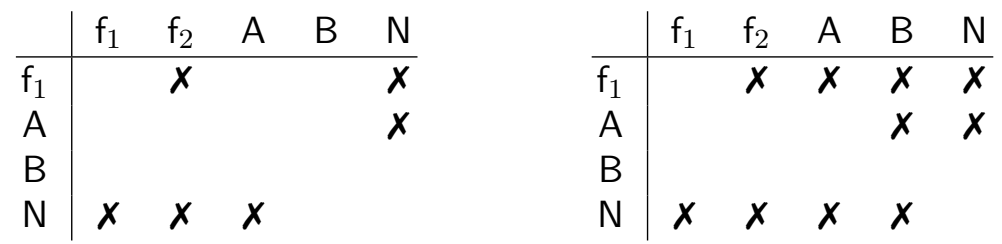

The left matrix determines unification compatibility, while the right matrix determines compatibility for matching term $s$ (rows) onto term $t$ (columns). Symbols $\mathrm{f}_{1}$ and $\mathrm{f}_{2}$ stand for arbitrary distinct constants. Incompatible features are marked with $\boldsymbol{X}$. For example, given a tuple of term positions $(1,1.1 .1,2)$, and terms $f(g(X), b)$ and $f(f(a, a), b)$, their fingerprints are $(g, B, b)$ and $(f, N, b)$, respectively. Since the first fingerprint component is incompatible, terms are not unifiable.

Fingerprints for the terms in the index are stored in a trie data structure. This allows us to efficiently filter out terms that are not compatible with a given retrieval condition. For the remaining terms, a unification or matching procedure must be invoked to determine whether they satisfy the condition or not. 
The fundamental idea of first-order fingerprint indexing carries over to higher-order terms - application of a substitution does not change the rigid skeleton of a term. However, to extend fingerprint indexing to higher-order terms, we must address the issues of $\alpha \beta \eta$ normalization and figure how to cope with $\lambda$-abstractions and bound variables. To that end, we define a function $\lfloor t\rfloor$, defined on $\beta$-reduced $\eta$-long terms in De Bruijn [Bru75] notation:

$$
\lfloor F \bar{s}\rfloor=F \quad\left\lfloor x_{i} \bar{s}_{n}\right\rfloor=\mathrm{db}_{i}^{\alpha}\left(\left\lfloor s_{1}\right\rfloor, \ldots,\left\lfloor s_{n}\right\rfloor\right) \quad\left\lfloor\mathrm{f} \bar{s}_{n}\right\rfloor=\mathrm{f}\left(\left\lfloor s_{1}\right\rfloor, \ldots,\left\lfloor s_{n}\right\rfloor\right) \quad\lfloor\lambda \bar{x} . s\rfloor=\lfloor s\rfloor
$$

We let $x_{i}$ be a bound variable of type $\alpha$ with De Bruijn index $i$, and $\mathrm{db}_{i}^{\alpha}$ be a fresh constant corresponding to this variable. All constants $\mathrm{db}_{i}^{\alpha}$ must be fresh. Effectively, \lfloor\rfloor transforms a $\eta$-long $\beta$-reduced higher-order term to an untyped first-order term. Let $t_{\downarrow \beta \eta}$ be the $\eta$-long $\beta$-reduced form of $t$; the higher-order generic fingerprinting function gfpf $_{\text {ho }}$, which relies on conversion $\langle t\rangle_{\mathrm{db}}$ from named to De Bruijn representation, is defined as

$$
\operatorname{gfpf}_{\text {ho }}(t, p)=\operatorname{gfpf}\left(\left\lfloor\left\langle t_{\downarrow \beta \eta}\right\rangle_{\mathrm{db}}\right\rfloor, p\right)
$$

If we define $\mathrm{fp}_{\mathrm{ho}}(t)=\mathrm{fp}\left(\left\lfloor\left\langle t_{\downarrow \beta \eta}\right\rangle_{\mathrm{db}}\right\rfloor\right)$, we can support fingerprint indexing for higher-order terms with no changes to the compatibility matrices. For example, consider the terms $s=(\lambda x y . x y) \mathrm{g}$ and $t=\mathrm{f}$, where $\mathrm{g}$ has the type $\alpha \rightarrow \beta$ and $\mathrm{f}$ has the type $\alpha \rightarrow \alpha \rightarrow \beta$. For the tuple of positions $(1,1.1 .1,2)$ we get

$$
\begin{aligned}
& \mathrm{fp}_{\mathrm{ho}}(s)=\mathrm{fp}\left(\left\lfloor\left\langle s_{\downarrow \beta \eta}\right\rangle_{\mathrm{db}}\right\rfloor\right)=\mathrm{fp}\left(\mathrm{g}\left(\mathrm{db}_{0}^{\alpha}\right)\right)=\left(\mathrm{db}_{0}^{\alpha}, \mathrm{N}, \mathrm{N}\right) \\
& \mathrm{fp}_{\mathrm{ho}}(t)=\mathrm{fp}\left(\left\lfloor\left\langle t_{\downarrow \beta \eta}\right\rangle_{\mathrm{db}}\right\rfloor\right)=\mathrm{fp}\left(\mathrm{f}\left(\mathrm{db}_{1}^{\alpha}, \mathrm{db}_{0}^{\alpha}\right)\right)=\left(\mathrm{db}_{1}^{\alpha}, \mathrm{N}, \mathrm{db}_{0}^{\alpha}\right)
\end{aligned}
$$

Since the first and third fingerprint component are incompatible, the terms are not unifiable.

Other first-order indexing techniques such as feature vector indexing and substitution trees can probably be extended to higher-order terms using the method described here as well.

\section{IMPLEMENTATION}

Zipperposition [Cru15, Cru17] is an open-source ${ }^{4}$ theorem prover written in OCaml. It is a versatile testbed for prototyping extensions to superposition-based theorem provers. It was initially designed as a prover for polymorphic first-order logic and then extended to higher-order logic. A recent addition is a complete mode for Boolean-free higher-order logic $\left[\mathrm{BBT}^{+} 19\right]$, which depends on a unification procedure that can enumerate a CSU. We implemented our procedure in Zipperposition.

We used OCaml's functors to create a modular implementation. The core of our procedure is implemented in a module which is parametrized by another module providing oracles and implementing the Bind step. In this way we can obtain the complete or pragmatic procedure and seamlessly integrate oracles while reusing as much common code as possible.

To enumerate all elements of a possibly infinite CSU, we rely on lazy lists whose elements are subsingletons of unifiers (either one-element sets containing a unifier or empty sets). The search space must be explored in a fair manner, meaning that no branch of the constructed tree is indefinitely postponed.

Each Bind step will give rise to new unification problems $E_{1}, E_{2}, \ldots$ to be solved. Solutions to each of those problems are lazy lists $p_{1}, p_{2}, \ldots$ containing subsingletons of unifiers. To avoid postponing some unifier indefinitely, we use the dovetailing technique: we first take one subsingleton from $p_{1}$, then one from each of $p_{1}$ and $p_{2}$. We continue with one subsingleton from each of $p_{1}, p_{2}$ and $p_{3}$, and so on. Empty lazy lists are ignored in the

\footnotetext{
${ }^{4}$ https://github.com/sneeuwballen/zipperposition
} 
traversal. To ensure we do not remain stuck waiting for a unifier from a particular lazy list, the procedure will periodically return an empty set, indicating that the next lazy list should be probed.

The implemented selection function for our procedure prioritizes selection of rigid-rigid over flex-rigid pairs, and flex-rigid over flex-flex pairs. However, since the constructed substitution $\sigma$ is not applied eagerly, heads can appear to be flex, even if they become rigid after dereferencing and normalization. To mitigate this issue to some degree, we dereference the heads with $\sigma$, but do not normalize, and use the resulting heads for prioritization.

We implemented oracles for the pattern, solid, and fixpoint fragment. Fixpoint unification [Hue75] is concerned with problems of the form $\{F \stackrel{?}{=} t\}$. If $F$ does not occur in $t$, $\{F \mapsto t\}$ is an MGU for the problem. If there is a position $p$ in $t$ such that $\left.t\right|_{p}=F \bar{u}_{m}$ and for each prefix $q \neq p$ of $p,\left.t\right|_{q}$ has a rigid head and either $m=0$ or $t$ is not a $\lambda$-abstraction, then we can conclude that $F \stackrel{?}{=} t$ has no solutions. Otherwise, the fixpoint oracle is not applicable.

For second-order logic with only unary constants, it is decidable whether a unifier for a problem in this class (called monadic second-order) exists [Far88]. As this class of terms admits a possibly infinite CSU, this oracle cannot be used for OracleSucc step, but it can be used for OracleFail. Similarly the fragment of second-order terms with no repeated occurrences of free variables has decidable unifier existence but possibly infinite CSUs [Dow01]. Due to their limited applicability and high complexity we decided not to implement these oracles.

\section{Evaluation}

We evaluated the implementation of our unification procedure in Zipperposition, assessing the complete variant and the pragmatic variant, the latter with several different combinations of limits for number of bindings. As part of the implementation of the complete mode for Boolean-free higher-order logic in Zipperposition $\left[\mathrm{BBT}^{+} 19\right]$, Bentkamp implemented a straightforward version of the JP procedure. This version is faithful to the original description, with a check as to whether a (sub)problem can be solved using a first-order oracle as the only optimization. Our evaluations were performed on StarExec Miami [SST14] servers with Intel Xeon E5-2620 v4 CPUs clocked at $2.10 \mathrm{GHz}$ with 60 s CPU limit.

Contrary to first-order unification, there is no widely available corpus of benchmarks dedicated solely to evaluating performance of higher-order unification algorithms. Thus, we used all 2606 monomorphic higher-order theorems from the TPTP library [Sut17] and 832 monomorphic higher-order Sledgehammer $(\mathrm{SH})$ generated problems [SBP13] as our benchmarks ${ }^{5}$. Many TPTP problems require synthesis of complicated unifiers, whereas Sledgehammer problems are only mildly higher-order-many of them are solved with firstorder unifiers.

We used the naive implementation of the JP procedure (jp) as a baseline to evaluate the performance of our procedure. We compare it with the complete variant of our procedure (cv) and pragmatic variants (pv) with several different configurations of limits for applied bindings. All other Zipperposition parameters have been fixed to the values of a variant of a well-performing configuration we used for the CASC-27 theorem proving competition [Sut19]. The cv configuration and all of the pv configurations use only pattern unification as an

\footnotetext{
${ }^{5} \mathrm{An}$ archive with raw results, all used problems, and scripts for running each configuration is available at http://doi.org/10.5281/zenodo. 4269591
} 


\begin{tabular}{lrrrrrrrr} 
& \multicolumn{1}{c}{$\mathrm{jp}$} & \multicolumn{1}{c}{$\mathrm{cv}$} & $\mathrm{pv}_{6666}^{12}$ & $\mathrm{pv}_{3333}^{6}$ & $\mathrm{pv}_{2222}^{4}$ & $\mathrm{pv}_{1222}^{2}$ & $\mathrm{pv}_{1121}^{2}$ & $\mathrm{pv}_{1020}^{2}$ \\
\hline TPTP & 1551 & 1717 & 1722 & $\mathbf{1 7 3 2}$ & $\mathbf{1 7 3 2}$ & 1715 & 1712 & 1719 \\
SH & 242 & $\mathbf{2 6 0}$ & 253 & 255 & 255 & 254 & 259 & 257
\end{tabular}

Figure 1: Proved problems, per configuration

\begin{tabular}{lrrrrrrrr} 
& \multicolumn{1}{c}{$\mathrm{n}$} & $\mathrm{f}$ & $\mathrm{p}$ & \multicolumn{1}{c}{$\mathrm{s}$} & \multicolumn{1}{c}{$\mathrm{fp}$} & \multicolumn{1}{c}{ fs } & \multicolumn{1}{c}{$\mathrm{ps}$} & \multicolumn{1}{c}{ fps } \\
\hline TPTP & 1658 & 1717 & 1717 & 1720 & 1719 & $\mathbf{1 7 2 4}$ & 1720 & 1723 \\
SH & 245 & 255 & $\mathbf{2 6 0}$ & 259 & 255 & 254 & 258 & 254
\end{tabular}

Figure 2: Proved problems, per used oracle

underlying oracle. To test the effect of oracle choice, we evaluated the complete variant in 8 combinations: with no oracles $(\mathbf{n})$, with only fixpoint $(\mathbf{f})$, pattern $(\mathbf{p})$, or solid (s) oracle, and with their combinations: $\mathbf{f p}, \mathbf{f s}, \mathbf{p s}, \mathbf{f p s}$.

Figure 1 compares different variants of the procedure with the naive JP implementation. Each pv configuration is denoted by $\operatorname{pv}_{b c d e}^{a}$ where $a$ is the limit on the total number of applied bindings, and $b, c, d$, and $e$ are the limits of functional projections, eliminations, imitations, and identifications, respectively. Figure 2 summarizes the effects of using different oracles.

The configuration of our procedure with no oracles outperforms the JP procedure with the first-order oracle. This suggests that the design of the procedure, in particular lazy normalization and lazy application of the substitution, already reduces the effects of the JP procedure's main bottlenecks. Raw evaluation data shows that on TPTP benchmarks, complete and pragmatic configurations differ in the set of problems they solve - cv solves 19 problems not solved by $\mathrm{pv}_{2222}^{4}$, whereas $\mathrm{pv}_{2222}^{4}$ solves 34 problems $\mathrm{cv}$ does not solve. Similarly, comparing the pragmatic configurations with each other, $\mathrm{pv}_{3333}^{6}$ and $\mathrm{pv}_{2222}^{4}$ each solve 13 problems that the other one does not. The overall higher success rate of $\mathrm{pv}_{1020}^{2}$ compared to $\mathrm{pv}_{1222}^{2}$ suggests that solving flex-flex pairs by trivial unifiers often suffices for superposition-based theorem proving.

Counterintuitively, in some cases, using oracles can hurt the performance of Zipperposition. Using oracles typically results in generating smaller CSUs, whose elements are more general substitutions than the ones we obtain without oracles. These more general substitutions usually contain more applied variables, which Zipperposition's heuristics avoid due to their explosive nature. This can make Zipperposition postpone necessary inferences for too long. Configuration n benefits from this effect and therefore solves 18 TPTP problems that no other configuration in Figure 2 solves. The same effect also gives configurations with only one oracle an advantage over configurations with multiple oracles on some problems.

The evaluation sheds some light on how often solid unification problems appear in practice. The raw data show that configuration s solves 5 TPTP problems that neither $\mathrm{f}$ nor $\mathrm{p}$ solve. Configuration $\mathrm{f}$ solves $8 \mathrm{TPTP}$ problems that neither $\mathrm{s}$ nor $\mathrm{p}$ solve, while $\mathrm{p}$ solves 9 TPTP problems that two other configurations do not. This suggests that the solid oracle is slightly less beneficial than the fixpoint or pattern oracles, but still presents a useful addition the set of available oracles.

A subset of TPTP benchmarks, concerning operations on Church numerals, is designed to test the efficiency of higher-order unification. Our procedure performs exceptionally well on these problems - it solves all of them, usually faster than other competitive higher-order 


\begin{tabular}{lrrrrr} 
& CVC4 & Leo-III & Satallax & Vampire & Zipperposition $(\mathrm{cv})$ \\
\hline NUM020^1 & - & 0.46 & - & - & $\mathbf{0 . 0 3}$ \\
NUM021^1 & - & - & - & - & $\mathbf{4 . 1 0}$ \\
NUM415^1 $^{\wedge}$ & 45.80 & 0.34 & 0.21 & 0.42 & $\mathbf{0 . 0 3}$ \\
NUM416^1 $^{\wedge}$ & 47.37 & 0.92 & 0.21 & 0.41 & $\mathbf{0 . 0 7}$ \\
NUM417^1 $^{\wedge}$ & - & 49.73 & $\mathbf{0 . 3 0}$ & 0.40 & 0.45 \\
NUM418^1 $^{\wedge}$ & - & 0.40 & 1.29 & 0.38 & $\mathbf{0 . 0 3}$ \\
NUM419^1 & - & 0.42 & 23.33 & 0.37 & $\mathbf{0 . 0 3}$ \\
NUM798^1 & 46.29 & 0.35 & 4.01 & 0.38 & $\mathbf{0 . 0 3}$ \\
NUM799^1 & - & 5.05 & - & - & $\mathbf{0 . 0 3}$ \\
NUM800^1 & - & - & - & $\mathbf{0 . 3 7}$ & 3.15 \\
NUM801^1 & - & 0.73 & 38.77 & - & $\mathbf{0 . 5 0}$
\end{tabular}

Figure 3: Time needed to prove a problem, in seconds.

provers. There are 11 benchmarks in NUM category of TPTP that contain conjectures about Church numerals: NUM020^1, NUM021^1, NUM415^1, NUM416^1, NUM417^1, NUM418^1, NUM419^1, NUM798^1, NUM799^1, NUM800^1, and NUM801^1. We evaluated those problems using the same CPU nodes and the same time limits as above. In addition to Zipperposition, we used all higher-order provers that took part in the 2019 edition of CASC [Sut19] (in the THF category) for this evaluation: CVC4 1.7 [BCD ${ }^{+} 11$ ], Leo-III 1.4 [SB18], Satallax 3.4 [Bro12], Vampire 4.4 THF [KV13]. Figure 3 shows the CPU time needed to solve a problem or "-" if the prover timed out.

\section{Discussion and Related Work}

The problem addressed in this paper is that of finding a complete and efficient higherorder unification procedure. Three main lines of research dominated the research field of higher-order unification over the last forty years.

The first line of research went in the direction of finding procedures that enumerate CSUs. The most prominent procedure designed for this purpose is the JP procedure [JP76]. Snyder and Gallier [SG89] also provide such a procedure, but instead of solving flex-flex pairs systematically, their procedure blindly guesses the head of the necessary binding by considering all constants in the signature and fresh variables of all possible types. Another approach, based on higher-order combinators, is given by Dougherty [Dou93]. This approach blindly creates (partially applied) S-, K-, and I-combinator bindings for applied variables, which results in returning many redundant unifiers, as well as in nonterminating behavior even for simple problems such as $X \mathrm{a}=\mathrm{a}$.

The second line of research is concerned with enumerating preunifiers. The most prominent procedure in this line of research is Huet's [Hue75]. The Snyder-Gallier procedure restricted not to solve flex-flex pairs is a version of the PT procedure presented in Section 5 . It improves Huet's procedure by featuring a Solution rule.

The third line of research gives up the expressiveness of the full $\lambda$-calculus and focuses on decidable fragments. Patterns [Nip93] are arguably the most important such fragment in practice, with implementations in Isabelle [NPW02], Leo-III [SB18], Satallax [Bro12], $\lambda$ Prolog [MN12], and other systems. Functions-as-constructors [LM16] unification subsumes 
pattern unification but is significantly more complex to implement. Prehofer [Pre95] lists many other decidable fragments, not only for unification but also preunification and unifier existence problems. Most of these algorithms are given for second-order terms with various constraints on their variables. Finally, one of the first decidability results is Farmer's discovery [Far88] that higher-order unification of terms with unary function symbols is decidable.

Our procedure draws inspiration from and contributes to all three lines of research. Accordingly, its advantages over previously known procedures can be laid out along those three lines. First, our procedure mitigates many issues of the JP procedure. Second, it can be modified not to solve flex-flex pairs, and become a version of Huet's procedure with important built-in optimizations. Third, it can integrate any oracle for problems with finite CSUs, including the one we discovered.

The implementation of our procedure in Zipperposition was one of the reasons this prover evolved from proof-of-concept prover for higher-order logic to competitive higher-order prover. In the 2020 edition of CASC, Zipperposition won the higher-order division solving $84 \%$ of problems, which is 20 percentage points ahead of the runner-up.

\section{Conclusion}

We presented a procedure for enumerating a complete set of higher-order unifiers that is designed for efficiency. Due to a design that restricts the search space and a tight integration of oracles, it reduces the number of redundant unifiers returned and gives up early in cases of nonunifiability. In addition, we presented a new fragment of higher-order terms that admits finite CSUs. Our evaluation shows a clear improvement over previously known procedures.

In future work, we will focus on designing intelligent heuristics that automatically adjust unification parameters according to the type of the problem. For example, we should usually choose shallow unification for mostly first-order problems and deeper unification for hard higher-order problems. We plan to investigate other heuristic choices, such as the order of bindings and the way in which search space is traversed (breadth- or depth-first). We are also interested in further improving the termination behavior of the procedure, without sacrificing completeness. Finally, following the work of Libal [Lib15] and Zaionc [Zai85], we would like to consider the use of regular grammars to finitely present infinite CSUs. For example, the grammar $G::=\lambda x . x \mid \lambda x . \mathrm{f}(G x)$ represents all elements of the CSU for the problem $\lambda x . G(\mathrm{f} x) \stackrel{?}{=} \lambda x . \mathrm{f}(G x)$.

Acknowledgement. We are grateful to the maintainers of StarExec for letting us use their service. We thank Ahmed Bhayat, Jasmin Blanchette, Daniel El Ouraoui, Mathias Fleury, Pascal Fontaine, Predrag Janičić, Robert Lewis, Femke van Raamsdonk, Hans-Jörg Schurr, Sophie Tourret, Dmitriy Traytel, and the anonymous reviewers for suggesting many improvements to this text. Vukmirović and Bentkamp's research has received funding from the European Research Council (ERC) under the European Union's Horizon 2020 research and innovation program (grant agreement No. 713999, Matryoshka). Nummelin has received funding from the Netherlands Organization for Scientific Research (NWO) under the Vidi program (project No. 016.Vidi.189.037, Lean Forward). 


\section{REFERENCES}

$\left[\mathrm{BBT}^{+} 19\right]$ Alexander Bentkamp, Jasmin Blanchette, Sophie Tourret, Petar Vukmirović, and Uwe Waldmann. Superposition with lambdas. In Pascal Fontaine, editor, CADE-27, volume 11716 of LNCS, pages 55-73. Springer, 2019.

$\left[\mathrm{BCD}^{+} 11\right]$ Clark W. Barrett, Christopher L. Conway, Morgan Deters, Liana Hadarean, Dejan Jovanović, Tim King, Andrew Reynolds, and Cesare Tinelli. CVC4. In Ganesh Gopalakrishnan and Shaz Qadeer, editors, CAV 2011, volume 6806 of LNCS, pages 171-177. Springer, 2011.

[BR19] Ahmed Bhayat and Giles Reger. Restricted combinatory unification. In Pascal Fontaine, editor, CADE-27, volume 11716 of LNCS, pages 74-93. Springer, 2019.

[Bro12] Chad E. Brown. Satallax: An automatic higher-order prover. In Bernhard Gramlich, Dale Miller, and Uli Sattler, editors, IJCAR 2012, volume 7364 of LNCS, pages 111-117. Springer, 2012.

[Bru75] Nicolaas G. De Bruijn. Lambda calculus notation with nameless dummies, a tool for automatic formula manipulation, with application to the Church-Rosser theorem. J. Symb. Log., 40(3):470470, 1975.

[Cru15] Simon Cruanes. Extending Superposition with Integer Arithmetic, Structural Induction, and Beyond. PhD thesis, École polytechnique, 2015.

[Cru17] Simon Cruanes. Superposition with structural induction. In Clare Dixon and Marcelo Finger, editors, FroCoS 2017, volume 10483 of LNCS, pages 172-188. Springer, 2017.

[DM79] Nachum Dershowitz and Zohar Manna. Proving termination with multiset orderings. Commun. ACM, 22(8):465-476, 1979.

[Dou93] Daniel J. Dougherty. Higher-order unification via combinators. Theor. Comput. Sci., 114(2):273298, 1993.

[Dow01] Gilles Dowek. Higher-order unification and matching. In John Alan Robinson and Andrei Voronkov, editors, Handbook of Automated Reasoning, pages 1009-1062. Elsevier and MIT Press, 2001.

[Far88] William M. Farmer. A unification algorithm for second-order monadic terms. Ann. Pure Appl. Logic, 39(2):131-174, 1988.

[Hue75] Gérard P. Huet. A unification algorithm for typed lambda-calculus. Theor. Comput. Sci., 1(1):2757, 1975.

[HV09] Krystof Hoder and Andrei Voronkov. Comparing unification algorithms in first-order theorem proving. In Bärbel Mertsching, Marcus Hund, and Muhammad Zaheer Aziz, editors, KI 2009, volume 5803 of $L N C S$, pages 435-443. Springer, 2009.

[JP76] Don C. Jensen and Tomasz Pietrzykowski. Mechanizing omega-order type theory through unification. Theor. Comput. Sci., 3(2):123-171, 1976.

[KV13] Laura Kovács and Andrei Voronkov. First-order theorem proving and vampire. In Natasha Sharygina and Helmut Veith, editors, CAV 2013, volume 8044 of LNCS, pages 1-35. Springer, 2013.

[Lib15] Tomer Libal. Regular patterns in second-order unification. In Amy P. Felty and Aart Middeldorp, editors, CADE-25, volume 9195 of $L N C S$, pages 557-571. Springer, 2015.

[LM16] Tomer Libal and Dale Miller. Functions-as-constructors higher-order unification. In Delia Kesner and Brigitte Pientka, editors, FSCD 2016, volume 52 of LIPIcs, pages 26:1-26:17. Schloss Dagstuhl, 2016.

[LS16] Tomer Libal and Alexander Steen. Towards a substitution tree based index for higher-order resolution theorem provers. In Pascal Fontaine, Stephan Schulz, and Josef Urban, editors, PAAR 2016, volume 1635 of $C E U R$-WS, pages 82-94. CEUR-WS, 2016.

[MN12] Dale Miller and Gopalan Nadathur. Programming with Higher-Order Logic. Cambridge University Press, 2012.

[Nip93] Tobias Nipkow. Functional unification of higher-order patterns. In E. Best, editor, LICS '93, pages 64-74. IEEE Computer Society, 1993.

[NPW02] Tobias Nipkow, Lawrence C. Paulson, and Markus Wenzel. Isabelle/HOL - A Proof Assistant for Higher-Order Logic, volume 2283 of LNCS. Springer, 2002.

[Pre95] Christian Prehofer. Solving higher order equations: from logic to programming. PhD thesis, Technical University Munich, Germany, 1995.

[Rob65] John Alan Robinson. A machine-oriented logic based on the resolution principle. J. ACM, 12(1):2341, 1965. 
[SB18] Alexander Steen and Christoph Benzmüller. The higher-order prover Leo-III. In Didier Galmiche, Stephan Schulz, and Roberto Sebastiani, editors, IJCAR 2018, volume 10900 of $L N C S$, pages 108-116. Springer, 2018.

[SBP13] Nik Sultana, Jasmin Christian Blanchette, and Lawrence C. Paulson. LEO-II and Satallax on the Sledgehammer test bench. J. Applied Logic, 11(1):91-102, 2013.

[Sch12] Stephan Schulz. Fingerprint indexing for paramodulation and rewriting. In Bernhard Gramlich, Dale Miller, and Uli Sattler, editors, IJCAR 2012, volume 7364 of LNCS, pages 477-483. Springer, 2012.

[SG89] Wayne Snyder and Jean H. Gallier. Higher-order unification revisited: Complete sets of transformations. J. Symb. Comput., 8(1/2):101-140, 1989.

[SST14] Aaron Stump, Geoff Sutcliffe, and Cesare Tinelli. Starexec: A cross-community infrastructure for logic solving. In Stéphane Demri, Deepak Kapur, and Christoph Weidenbach, editors, IJCAR 2014, volume 8562 of $L N C S$, pages 367-373. Springer, 2014.

[Sut17] Geoff Sutcliffe. The TPTP problem library and associated infrastructure - from CNF to TH0, TPTP v6.4.0. J. Autom. Reasoning, 59(4):483-502, 2017.

[Sut19] Geoff Sutcliffe. The CADE-27 automated theorem proving system competition - CASC-27. AI Commun., 32(5-6):373-389, 2019.

[VBCS19] Petar Vukmirovic, Jasmin Christian Blanchette, Simon Cruanes, and Stephan Schulz. Extending a brainiac prover to lambda-free higher-order logic. In Tomás Vojnar and Lijun Zhang, editors, TACAS 2019, volume 11427 of LNCS, pages 192-210. Springer, 2019.

[VBN20] Petar Vukmirovic, Alexander Bentkamp, and Visa Nummelin. Efficient full higher-order unification. In FSCD, volume 167 of LIPIcs, pages 5:1-5:17. Schloss Dagstuhl - Leibniz-Zentrum für Informatik, 2020.

[Zai85] Marek Zaionc. The set of unifiers in typed lambda-calculus as regular expression. In Jean-Pierre Jouannaud, editor, RTA-85, volume 202 of $L N C S$, pages 430-440. Springer, 1985. 